OU-HET-211

June 1995

\title{
Motions of the String Solutions in the XXZ Spin Chain under a Varying Twist
}

\author{
N. Fumita, H. Itoyama and T. Oota ${ }^{\star}$ \\ Department of Physics, Graduate School of Science, \\ Osaka University, Toyonaka, Osaka 560, Japan
}

\begin{abstract}
* This work is supported in part by a Grant-in-Aid for Scientific Research (07640403) from the Ministry of Education, Japan. e-mail: itoyama@funpth.phys.sci.osaka-u.ac.jp toota@funpth.phys.sci.osaka-u.ac.jp
\end{abstract}


We determine the motions of the roots of the Bethe ansatz equation for the ground state in the XXZ spin chain under a varying twist angle. This is done by analytic as well as numerical study at a finite size system. In the attractive critical regime $0<\Delta<1$, we reveal intriguing motions of strings due to the finite size corrections to the length of the strings: in the case of two-strings, the roots collide into the branch points perpendicularly to the imaginary axis, while in the case of three- strings, they fluctuate around the center of the string. These are successfully generalized to the case of $n$-string. These results are used to determine the final configuration of the momenta as well as that of the phase shift functions. We obtain these as well as the period and the Berry phase also in the regime $\Delta \leq-1$, establishing the continuity of the previous results at $-1<\Delta<0$ to this regime. We argue that the Berry phase can be used as a measure of the statistics of the quasiparticle ( or the bound state) involved in the process. 


\section{Introduction}

The one-dimensional spin one-half XXZ chain with the Hamiltonian

$$
H=-\frac{1}{2} \sum_{j=1}^{N}\left(\sigma_{j}^{x} \sigma_{j+1}^{x}+\sigma_{j}^{y} \sigma_{j+1}^{y}+\Delta \sigma_{j}^{z} \sigma_{j+1}^{z}\right)
$$

provides a soluble many-body problem which has been intensively studied for more than two decades and is the subject of the present paper. Here $\sigma_{j}^{x, y, z}$ are the Pauli matrices at site $j$ and $\Delta$ denotes the parameter of anisotropy. Let a generic M-body state of the model be represented by

$$
|\chi\rangle=\sum_{x_{1}=1}^{N} \cdots \sum_{x_{M}=1}^{N} \chi\left(x_{1}, \ldots, x_{M}\right) \prod_{j=1}^{M} \sigma_{x_{j}}^{-}|\uparrow\rangle
$$

where $|\uparrow\rangle$ is the state with all spins up and $\sigma_{x}^{-}$is the spin-lowering matrix at site $x$. The eigenfunction $\chi\left(x_{1}, \ldots, x_{M}\right)$ is of the form proposed by Bethe [1]:

$$
\begin{aligned}
& \chi\left(x_{1}, \ldots, x_{M} \mid p_{1}, \ldots, p_{M}\right) \\
= & {\left[\prod_{M \geq b>a \geq 1} \epsilon\left(x_{b}-x_{a}\right)\right] \sum_{Q}(-1)^{|Q|} \exp \left[i \sum_{a=1}^{M} x_{a} p_{Q a}+\frac{i}{2} \sum_{M \geq b>a \geq 1} \theta\left(p_{Q b}, p_{Q a}\right) \epsilon\left(x_{b}-x_{a}\right)\right] }
\end{aligned}
$$

where $\epsilon(x)$ is the sign function, the summation is with respect to permutations $Q$ of the momenta $p$ and $\theta$ is the two-particle phase shift. For two particles with momenta $p$ and $q$, the explicit form of the phase shift is $\theta(p, q)=2 \arctan \left[\frac{\Delta \sin [(p-q) / 2]}{\cos [(p+q) / 2]-\Delta \cos [(p-q) / 2]}\right]$. The energy of this eigenstate is found to be $E=-\frac{N}{2} \Delta+2 \sum_{j=1}^{M}\left(\Delta-\cos p_{j}\right)$. The second term is the sum of the single-particle energies $\varepsilon_{j}=2\left(\Delta-\cos p_{j}\right)$. The operator $M=\sum_{j=1}^{N} \frac{1}{2}\left(1-\sigma_{j}^{z}\right)$ is conserved and the case of half-filling corresponds

* We find it impossible to exhaust all references on this subject. The partial list includes [1]-[12]. The developments in recent years include the relation to the study of finite size corrections as well as the approach based on the affine $U_{q}(\hat{s l}(2))$, which are seen, for example, in [8], [9], [11], [12]. 
to $M=N / 2$. The complete reducibility of the problem to the two-body problem with the dynamical phase shift as well as the rigorous notion of the quasiparticle excitations is the hallmark of the integrable quantum systems whose mathematical structure is captured by the Yang-Baxter relation [13] [14] and the attendant quantum inverse scattering formalism [15].

The boundary condition is a heart to this problem as this determines the spectra of the model and is referred to as the Bethe ansatz equation in this paper. Let us now impose, contrary to the standard periodic boundary condition, the twisted boundary condition

$$
\chi\left(x_{1}, \ldots, x_{j}+N, \ldots, x_{M}\right)=\mathrm{e}^{i \Phi} \chi\left(x_{1}, \ldots, x_{j}, \ldots, x_{M}\right),
$$

where $\Phi$ is a twist angle. This leads to the system of transcendental equations for the set of momenta $\left\{p_{j}\right\}$

$$
N p_{j}+\sum_{k=1(\neq j)}^{M} \theta\left(p_{j}, p_{k}\right)=2 \pi I_{j}+\Phi
$$

where $j=1, \ldots, M$. We denote by $\left\{I_{j}\right\}$ the set of half-integers for even $M$ or integers for odd $M$.

In this paper, we study ground state properties of the system when the twist angle $\Phi$ is varied. The change of an external parameter like $\Phi$ in integrable systems will correspond to an adiabatic change in more general systems which do not possess an infinite number of conservation laws . The process we consider may be called adiabatic process in this sense. The major purpose of this paper is to examine the motions of the roots of eq. (1.5)under this process and to determine the momenta and the phase shift functions for the final configuration, given the ones for the initial configuration.

$\dagger$ The twist angle plays an important role in projecting out some states to construct another model. See [10] [16] for example. 
It is well-known that the system can be represented by the Jordan-Wigner fermions. The state with spin-up is regarded as an unoccupied state and the state with spin-down as an occupied state. The annihilation operator $C_{j}$ and the creation operator $C_{j}^{\dagger}$ are respectively defined by $C_{j}=\frac{1}{2}\left(\sigma_{j}^{x}+i \sigma_{j}^{y}\right) \prod_{k<j} \sigma_{k}^{z}, C_{j}^{\dagger}=$ $\frac{1}{2}\left(\sigma_{j}^{x}-i \sigma_{j}^{y}\right) \prod_{k<j} \sigma_{k}^{z}$, which satisfy anticommutation relations $\left\{C_{j}, C_{k}^{\dagger}\right\}=\delta_{j, k}$. The Hamiltonian (1.1) is rewritten in terms of lattice fermions as

$$
H=-\sum_{j}\left(C_{j}^{\dagger} C_{j+1}+C_{j+1}^{\dagger} C_{j}\right)-2 \Delta \sum_{j}\left(C_{j}^{\dagger} C_{j}-\frac{1}{2}\right)\left(C_{j+1}^{\dagger} C_{j+1}-\frac{1}{2}\right) .
$$

The twisted boundary condition can be attributed to the twisting of the operators $C_{j}=\mathrm{e}^{i \Phi} C_{j+N}, \quad C_{j}^{\dagger}=\mathrm{e}^{-i \Phi} C_{j+N}^{\dagger}$. The conserved operator $M=\sum_{j=1}^{N} C_{j} C_{j}^{\dagger}$ measures the fermion number. The twisted boundary condition is related to the Aharonov-Bohm effect if we consider the $M$ fermions carrying a charge $(-q)$ on the ring threaded by the magnetic flux $\Phi / q$ : as each fermion goes around the ring, it picks up the Aharonov-Bohm phase $\Phi$.

The change of the twist angle $\Phi$ is equivalent to varying the magnetic flux penetrating the ring which generates an electric field around the ring. The whole spectrum of Hamiltonian is periodic with respect to $\Phi$ with period $2 \pi$. If we follow each individual energy level, this is not necessarily the case however. The period of the ground state, in particular, is a nontrivial quantity to be determined. In the case of no interaction $\Delta=0$, the period can be obtained by a classical consideration alone. All negative energy pseudoparticles are accelerated in the electric field $E$ generated by the change of the flux $\Delta \Phi$ through $\Delta \Phi=\Phi\left(t_{I}\right)-$ $\Phi\left(t_{F}\right)=\int_{t_{F}}^{t_{I}} d t \oint d x q E$. The change of the momentum is $\Delta p_{j}=\int_{t_{I}}^{t_{F}} d t q E=\frac{\Delta \Phi}{N}$ where $N=\oint d x$. Thus the period of the ground state is $\Delta \Phi=2 N \pi$. In this process all negative energy pseudoparticle move from the first Brillouin zone $-\pi<p<\pi$ to the second Brillouin zone $\pi<p<3 \pi$.

A completely different picture is known to be obtained as soon as the the interaction is turned on [17]. In [17], the regime $-1<\Delta<0$ is considered. It is found that only one negative energy pseudoparticle moves from the first Brillouin 
zone $-\pi<p<\pi$ to the second Brillouin zone $\pi<p<3 \pi$. The period of the ground state is found to be $\Delta \Phi=4 \pi$. During this process a level crossing occurs. The ground state becomes the excited state for the system with the original periodic boundary condition at $\Phi=2 \pi$ and comes back to the original ground state at $\Phi=4 \pi$. The Berry phase attendant with this process has been considered in ref. [18] and is found to be $\pi$.

In the regime $0<\Delta<1$, more interesting events take place. It is pointed out in ref. [19] that, in the region $\cos (\pi / n+1)>\Delta>\cos (\pi / n)$, a bound state of the $n$-string is formed during the process. Only these roots of the Bethe ansatz equation forming the $n$-string move from the first Brillouin zone $-\pi<p<\pi$ to the second Brillouin zone $\pi<p<3 \pi$. The period of the ground state is again found to be $\Delta \Phi=4 \pi$.

The thrusts of the present paper consist of the following several points. In section 2 , we consider the region $0<\Delta<1$. In the segment $\cos (\pi / n+1)>$ $\Delta>\cos (\pi / n)$ for $n=2,3, \cdots$, we determine the motions of the roots of the Bethe ansatz eq. (1.5)in the rapidity plane ${ }^{\star}$. This is done on the basis of our numerical and analytic study at a finite size system. Here we find intriguing motions of the $n$-string during the process. In order to state our results here more clearly, let us recall that the $n$-string is a group of rapidities which differ in their imaginary parts by $2 i(\pi-\mu)$ and center on the $i \pi$ line $(\operatorname{Im} \lambda=\pi)$ :

$$
\begin{gathered}
\lambda_{k}^{(n)}=\left[\lambda^{(n)}+i \pi\right]+i(\pi-\mu)(n+1-2 k)+\delta_{k}^{(n)} \\
k=1, \ldots, n
\end{gathered}
$$

where $\left[\lambda^{(n)}+i \pi\right]$ is the center of the $n$-string and $\delta_{k}^{(n)}$ is a deviation due to the finiteness of the system from the string found in the infinite volume limit. This deviation is at most of order $1 / N$. We find, however, that it is this deviation which

\footnotetext{
* In this paper, the words "root" and "rapidity" are used interchangeably.

$\dagger$ In this paper, we deal with a natural formation of the $n$-string configuration. The Takahashi condition [6] will be automatically satisfied.
} 
plays a vital role in determining the global structure of the motion of the roots under the process. To be more explicit, we find that, in the case of the two-string, the roots collide into the branch points of the momenta and those of the phase shift functions perpendicularly to the imaginary axis. In the case of the threestring, we find that the roots fluctuate around the center of the string due to the finite size correction $\delta_{k}^{(n)}$ mentioned above. The case of the $n$-string is found to a generalization of these: for $n$ odd, the roots oscillate around the center of the string while, for $n$ even, in addition to the fluctuation, the two roots in the middle collide into the branch points. This fluctuation of string state creates a winding around the cut of the momentum and the phase shift function and determines the final configurations of these. These are presented in section 2 .

In section 3 , we consider the regime $\Delta \leq-1$ and determine the motion of the roots. From this we determine the period, the final configurations of the momenta and the phase shift functions and the Berry phase. These values are found to be the same as those derived in ref.[17], [18] in $-1<\Delta<0$. We, therefore, establish the continuity at $\Delta=-1$.

In section 4 , We compute the Berry phase in the regime $0<\Delta<1$ and consider the physics associated with it. By piecing through the computation done in [18] and ours, we are led to propose that the Berry phase provides a measure of the statistics of the quasiparticle (or the bound state) involved in the process. We consider statistics of particles as the Berry phase arising through $p$-space monodromy.

In Appendix A, we summarize our analytic study of the finite size system at $M=2, N=4$. In Appendix B and Appendix C, we present proofs of technical lemmas necessary in $\S 2-1$ and $\S 4$ respectively. 


\section{Motions of String Solutions under a Varying Twist Angle in the Regime $0<\Delta<1$}

In the regime $-1<\Delta<1$, the momentum $p$, the phase shift $\theta$ and the total energy $E$ are parametrized by rapidities $\lambda_{j}$;

$$
\begin{gathered}
p(\lambda)=-i \ln \left[-\frac{\sinh \frac{1}{2}(\lambda-i \mu)}{\sinh \frac{1}{2}(\lambda+i \mu)}\right], \\
\theta\left(\lambda_{1}, \lambda_{2}\right)=i \ln \left[-\frac{\sinh \frac{1}{2}\left(\lambda_{1}-\lambda_{2}-2 i \mu\right)}{\sinh \frac{1}{2}\left(\lambda_{1}-\lambda_{2}+2 i \mu\right)}\right], \\
E=\frac{N}{2} \cos \mu+\sum_{j=1}^{M} \frac{-2 \sin ^{2} \mu}{\cosh \lambda_{j}-\cos \mu},
\end{gathered}
$$

where $\Delta=-\cos \mu$. Let us imagine a closed circuit in the $\lambda$ plane consisting of the real axis and the $i \pi$ line connected at plus and minus infinity. The momentum $p(\lambda)$ increases monotonically as a function of $\lambda$ from $-(\pi-\mu)$ at $\lambda=-\infty$ to $(\pi-\mu)$ at $\lambda=\infty$. ( See Figure 2.1). If $p$ increases further, $\lambda$ moves backward along the $i \pi$ line from $+\infty+i \pi$. The momentum $p(\lambda)$ varies from $(\pi-\mu)$ at $\lambda=\infty+i \pi$ to $2 \pi-(\pi-\mu)$ at $\lambda=-\infty+i \pi$. When the rapidity goes around this closed circuit, the momentum $p$ increases by $2 \pi$, since the path encircles a logarithmic branch cut as a function of $\lambda$. We note that the single-particle energy

$$
\varepsilon_{j}=\frac{-2 \sin ^{2} \mu}{\cosh \lambda_{j}-\cos \mu}
$$

is negative for $\operatorname{Im} \lambda_{j}=0$ and positive for $\operatorname{Im} \lambda_{j}=\pi$.

The behavior of the phase shift function $\theta(\lambda)$ depends on the sign of $\Delta$. ( See Figure 2.1.) When $\Delta<0\left(0<\mu<\frac{\pi}{2}\right)$, the phase shift $\theta(\lambda)$ decreases from $(\pi-2 \mu)$ at $\lambda=-\infty$ to $-(\pi-2 \mu)$ at $\lambda=\infty$. The phase shift further decreases from $-(\pi-2 \mu)$ at $\lambda=\infty+i \pi$ to $-2 \pi+(\pi-2 \mu)$ at $\lambda=-\infty+i \pi$. As the 
relative rapidity goes around the circuit, the phase shift decreases by $2 \pi$. When $\Delta>0\left(\frac{\pi}{2}<\mu<\pi\right)$, the phase shift $\theta(\lambda)$ increases from $-(2 \mu-\pi)$ at $\lambda=-\infty$ to $(2 \mu-\pi)$ at $\lambda=\infty$. The phase shift further increases from $(2 \mu-\pi)$ at $\lambda=\infty+i \pi$ to $2 \pi-(2 \mu-\pi)$ at $\lambda=-\infty+i \pi$. As the relative rapidity goes around this circuit, the phase shift increases by $2 \pi$. This difference plays a central role in determining the behavior of the rapidities as seen later.

Before we study the region $0<\Delta<1$, we briefly look at the region $-1<\Delta<0$ considered in ref.[17]. All the rapidities are symmetrically arranged on the real axis at $\Phi=0$. We denote them by $-\infty<\lambda_{1}<\lambda_{2}<\cdots<\lambda_{M}<\infty$. The largest rapidity $\lambda_{M}$ goes to infinity at $\Phi=2(\pi-\mu)$. Here the $\lambda_{M}$ jumps from the real axis where a negative-energy mode lies to the $i \pi$ line where a positive-energy mode lies. This means a particle creation. When $\Phi$ increases from $2(\pi-\mu), \lambda_{M}$ moves backward along the $i \pi$ line and the other rapidities remain on the real axis. The $\lambda_{M}$ on $i \pi$ line may be called one-string. When $\Phi=2 \pi$, the rapidity $\lambda_{M}$ reaches $i \pi$. When $\Phi=2 \pi-2(\pi-\mu), \lambda_{M}$ goes to $-\infty+i \pi$ and jumps onto the real axis. This means a particle annihilation. The state comes back to the original ground state at $\Phi=4 \pi$. The relation between the initial and the final momentum and the one between the initial and the final phase shift are the same as in the regime $\Delta<-1$, which we will show in $\S 4$. The Berry phase of the ground state wave function in this regime has been calculated in ref.[18] to be $\pi$.

In the case of half-filling $M=N / 2$ which we consider, the picture of the Dirac sea emerges as follows. The eq. (1.5) reduces to $p_{j}=\left(I_{j} / N\right) \times 2 \pi$ with the condition $\Phi=0$. The single-particle energy is $\varepsilon_{j}=-2 \cos p_{j}=-2 \cos \left(2 \pi I_{j} / N\right)$. The negative energy modes are in the region $\left|I_{j}\right| \leq N / 4$ and are given by the set

$$
I_{j}=-\frac{M-1}{2},-\frac{M-3}{2}, \ldots, \frac{M-1}{2} .
$$

The same set $\left\{I_{j}\right\}$ turns out to provide the ground state of the half-filling sector in the interacting case. 


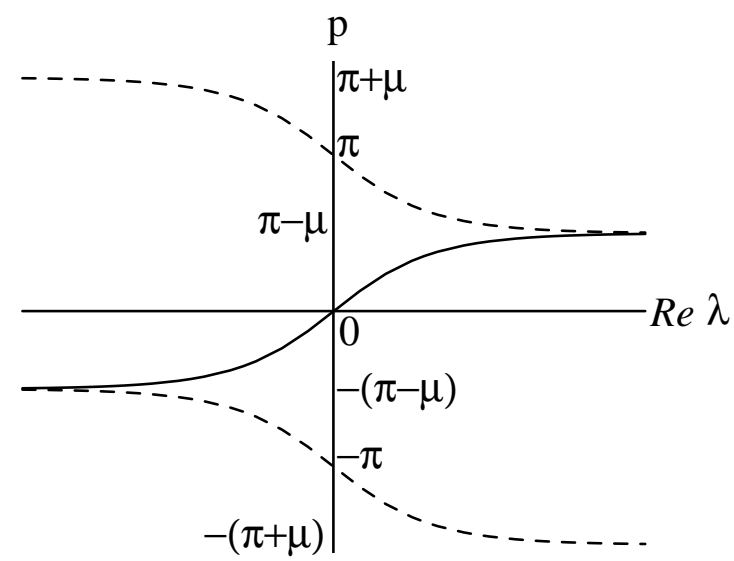

(a) momemtum

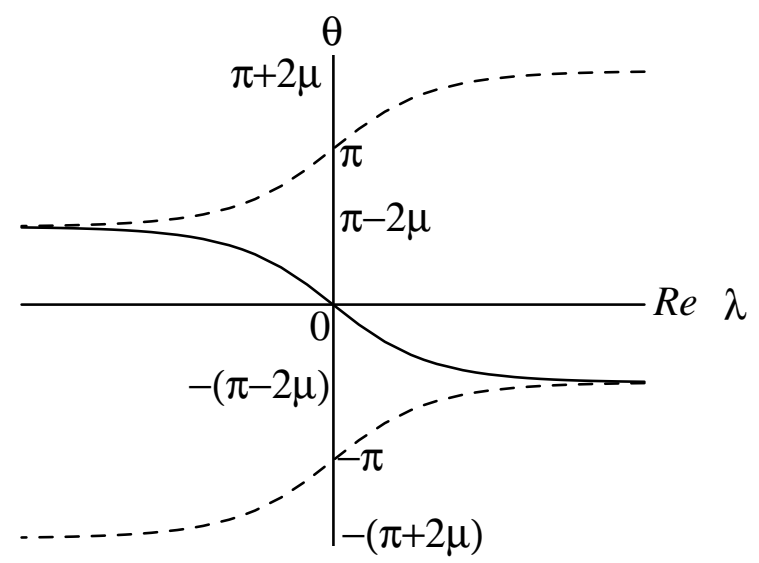

(b) phase-shift $(0<\mu<\pi / 2)$

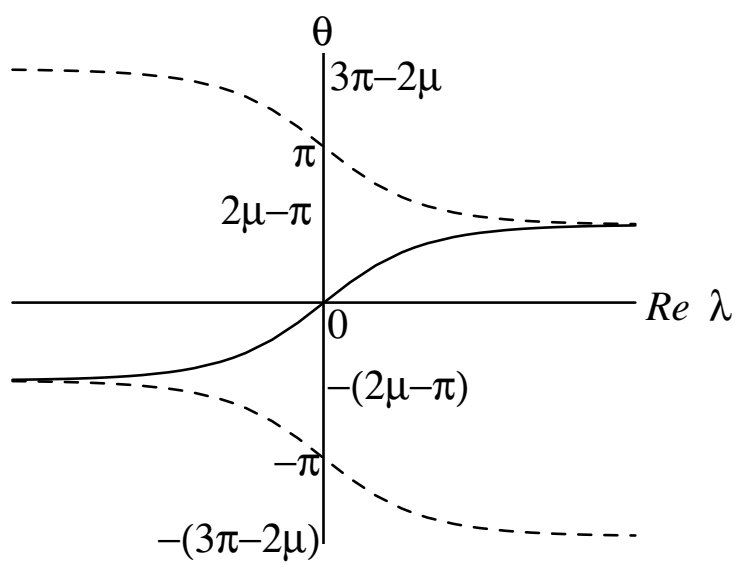

(c) phase-shift $(\pi / 2<\mu<\pi)$

Figure 2-1. The momentum function and the phase-shift function for $\operatorname{Im} \lambda=0$ (normal lines) and for $\operatorname{Im} \lambda=\pi$ (dotted lines).

In the next two subsections, we consider the case in which only a two-string is formed $(0<\Delta<\cos (\pi / 3))$ and the case in which a three-string is formed $(\cos (\pi / 3)<\Delta<\cos (\pi / 4))$. The conclusion we will draw in the former case is mainly based on the analytic study we have made at a finite size system $M=$ 
$2, N=4$, which we present in Appendix A. The conclusion we will draw in the latter case is originally derived from the numerical study we have carried out in the case $M=3, N=6$. In both cases, we check the consistency condition that the momenta and the phase shift functions for the final configuration follow from those for the initial one by using the relations we derive from the global behavior of the rapidities. We find that this consistency condition is a nontrivial check to make. In the last subsection, we are able to see that the global behavior of the rapidities we find in the case of $n$-string formation $(\cos (\pi / n)<\Delta<\cos (\pi / n+1))$ passes this consistency check for any $n$. This check is very stringent and consolidates our conclusion.

It will be helpful to point out here that one can predict a few things about the behavior of the rapidities without looking at the details of eq. (1.5). As we vary the twist angle continuously, an individual momentum, which is a root of eq. (1.5), must vary continuously. The only place in which a set of rapidities can jump to form a string while keeping the momenta continuous is the point at infinity ( i.e. the real part of the rapidity is infinite. See eq. (2.1)). This is also the place for the formation of a zero-energy bound state. ( See eq. (2.4).) The value of the twist angle where the formation of $n$-string at infinity can occur can be obtained from eqs. (1.5), (2.1). We start from eq. (1.5)for $M$ rapidities and send the largest ( or smallest) $n$ rapidities to plus ( or minus ) infinity, which subsequently form an $n$-string. Only when the twist angle $\Phi=+($ or -$) n(\pi-\mu)$, the rest of the $M-n$ rapidities are kept symmetric with respect to the origin, staying in the ground state configuration. In other words, only at this value the ground state configuration for $M$ rapidities reduces to that for the $M-n$ rapidities.

\subsection{GROUND STATE IN THE CASE OF TWO STRING}

We now study the more specific case $0<\Delta<\cos (\pi / 3)\left(\frac{\pi}{2}<\mu<\frac{2}{3} \pi\right)$ where the two-string is expected to be formed during the process. For convenience we describe the process to be associated with the change of $\Phi$ from $-2 \pi$ to $2 \pi$. We first examine the latter half $0 \leq \Phi<2 \pi$ of the process. When $\Phi$ increases from 
zero the largest rapidity $\lambda_{M}$ reaches $\infty$ at $\Phi=2(\pi-\mu)$ just as in the $-1<\Delta<0$ case. The rapidity $\lambda_{M}$ jumps to the $i \pi$ line and begins to move backward along the $i \pi$ line from $\infty+i \pi$. The dramatic change arises when $\Phi$ increases further. The rapidity $\lambda_{M}$ on the $i \pi$ line returns to $\infty+i \pi$. The second largest rapidity $\lambda_{M-1}$ on the real axis goes to infinity. The two rapidities $\lambda_{M}$ and $\lambda_{M-1}$ attract each other. At $\Phi=4(\pi-\mu)$, the two rapidities reach $\lambda_{M}=\infty+i \pi$ and $\lambda_{M-1}=\infty$. The two largest rapidities then jump together by $i \pi / 2$ to the imaginary direction and they form a two-string: $\lambda_{M}=\infty+\frac{3}{2} i \pi$ and $\lambda_{M-1}=\infty+\frac{1}{2} i \pi$. This means a formation of the bound state over the vacuum. The two rapidities are separated by $i \pi$ and their center is on the $i \pi$ line. So the deviation due to the finite size in (1.7) is given by $\delta_{M}=\delta_{1}^{(2)}=i \mu$ and $\delta_{M-1}=\delta_{2}^{(2)}=-i \mu$.

When we further increase $\Phi$, the two-string moves backward from plus infinity. The center moves on the $i \pi$ line from $\infty+i \pi$. In this process the difference between $\lambda_{M}$ and $\lambda_{M-1}$ decreases from $i \pi$. At $\Phi=2 \pi$, the two-string arrives at the imaginary axis:

$$
\begin{aligned}
\lambda_{M} & =i(2 \pi-\mu), \\
\lambda_{M-1} & =i \mu
\end{aligned}
$$

We note that the relative rapidity $\left(\lambda_{M}-\lambda_{M-1}\right)$ is $2 i(\pi-\mu)$, and the deviations $\delta_{1}^{(2)}$ and $\delta_{2}^{(2)}$ in (1.7) vanishes. It is interesting that the momenta $p\left(\lambda_{M}\right), p\left(\lambda_{M-1}\right)$ and the phase shift $\theta\left(\lambda_{M}, \lambda_{M-1}\right)$ are divergent at this point. The points (2.6) are the branch points of both the momentum $p(\lambda)$ and the phase shift $\theta(\lambda)$, which are logarithmic functions (2.1) and (2.2) respectively. If the rapidity $\lambda_{j}$ goes around the branch point $\lambda=i \mu(\lambda=i(2 \pi-\mu))$ counterclockwise, the momentum $p\left(\lambda_{j}\right)$ increases (decreases) by $2 \pi$. If the relative rapidity $\lambda$ goes around the branch point $\lambda=2 i(\pi-\mu)$ counterclockwise, the phase shift increases by $2 \pi$. Therefore the behavior of the rapidities around these branch points has a global meaning and is necessary to determine the final configuration of the system. The paths of the rapidities $\lambda_{M}, \lambda_{M-1}$ and those of the relative rapidity $\lambda_{M}-\lambda_{M-1}$ around the branch points are plotted in Fig. 2-2 and Fig. 2-3 respectively. The $\lambda_{M}$ and $\lambda_{M-1}$ collide into the singularities perpendicularly to the imaginary axis (Fig. 2-2). The 
value of the relative rapidity $\lambda_{M}-\lambda_{M-1}$ reduces from $i \pi$ to $2 i(\pi-\mu)$ (Fig. 2-3). This behavior of the rapidities is checked both by the numerical calculation and by the analytic calculation in the case of $M=2$ which is described in Appendix A. The first half process in $-2 \pi<\Phi \leq 0$ is similar to the latter half process. At $\Phi=-2 \pi$, the two-string starts from the singular points $\lambda_{1}=i(2 \pi-\mu), \lambda_{2}=i \mu$. When $\Phi=-4(\pi-\mu)$ the two-string reaches $\lambda_{1}=-\infty+i \frac{3}{2} \pi$ and $\lambda_{2}=-\infty+i \frac{\pi}{2}$. After jumping by $-i \pi / 2, \lambda_{1}$ and $\lambda_{2}$ move on the $i \pi$ line and the real axis respectively. Finally $\lambda_{1}$ jumps onto the real axis at $\Phi=-2(\pi-\mu)$.

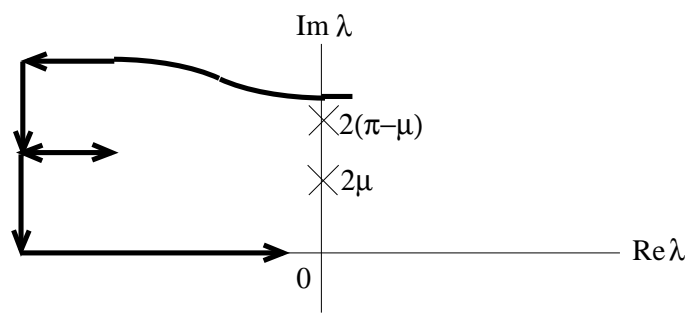

(a) the path of $\lambda_{1}$

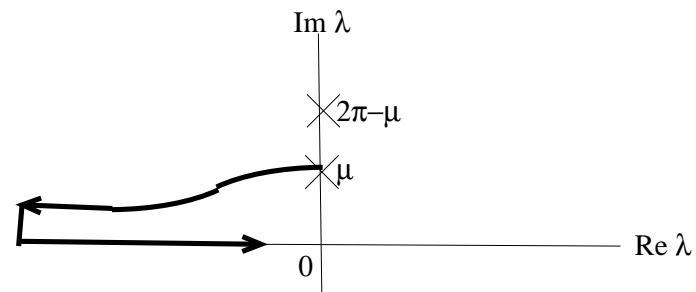

(c) the path of $\lambda 2$

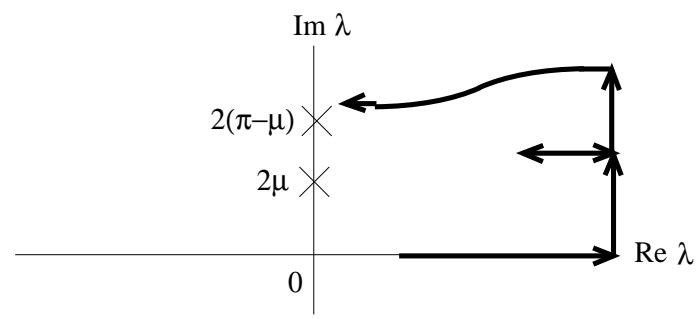

(b) the path of $\lambda_{\mathrm{M}}$

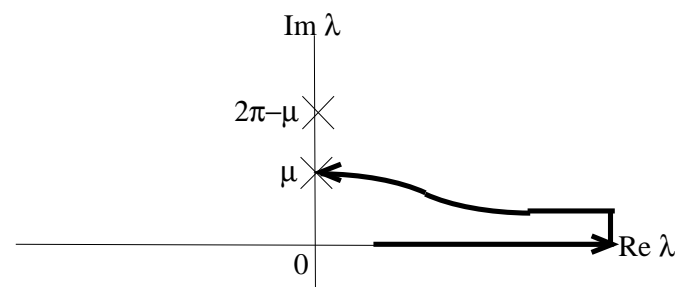

(d) the path of $\lambda_{M-1}$

Figure 2-2. The paths of the rapidities in case of $\pi / 2<\mu<2 \pi / 3$.

The relations between the initial state $(\Phi=-2 \pi)$ and the final state $(\Phi=2 \pi)$ 


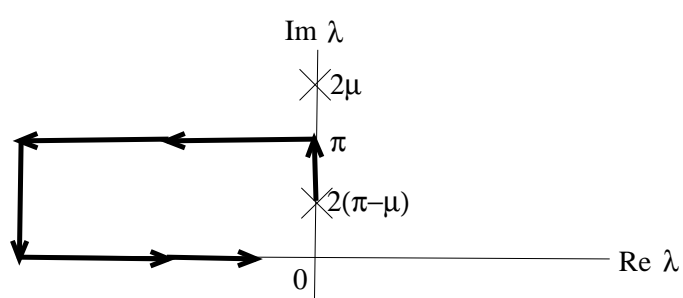

(a) the path of $\left(\lambda_{1}-\lambda_{2}\right)$

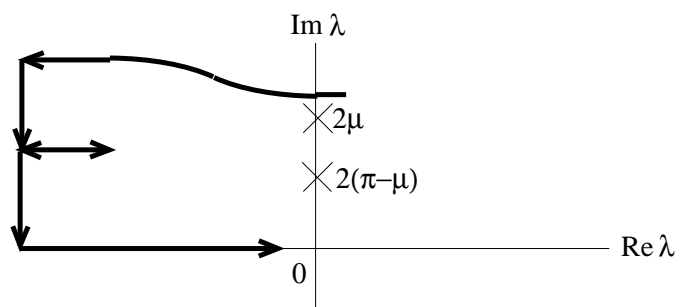

(c) the path of $\left(\lambda_{1}-\lambda_{j+2}\right)$ $(\mathrm{j}=1,2, \ldots \mathrm{M}-2)$

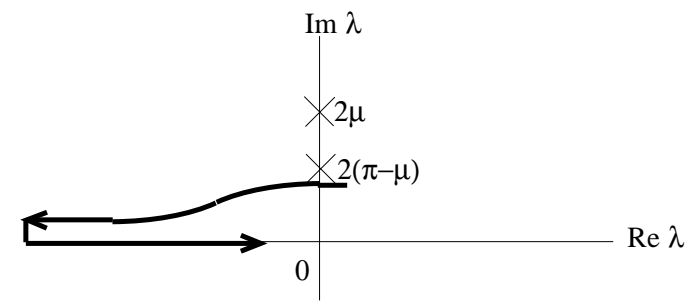

(e) the path of $\left(\lambda_{2}-\lambda_{j+2}\right)$

$(\mathrm{j}=1,2, \ldots \mathrm{M}-2)$

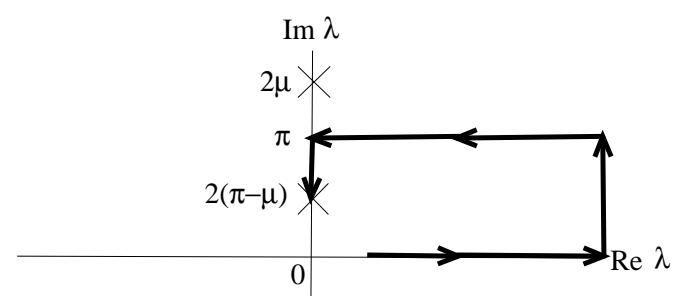

(b) the path of $\left(\lambda_{M}-\lambda_{M-1}\right)$

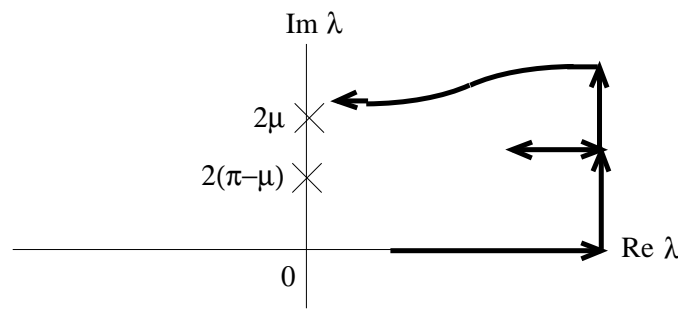

(d) the path of $\left(\lambda_{M}-\lambda_{j}\right)$

$(\mathrm{j}=1,2, \ldots \mathrm{M}-2)$

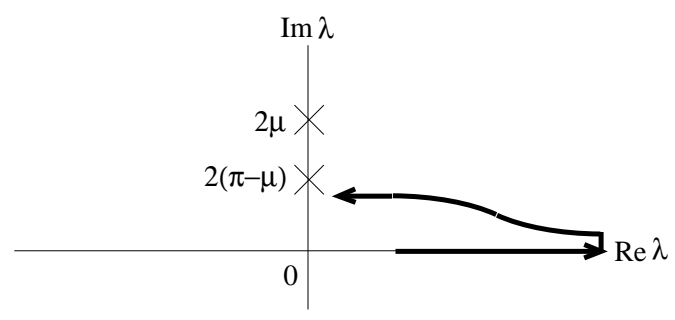

(f) the path of $\left(\lambda_{M-1}-\lambda_{j}\right)$

$(\mathrm{j}=1,2, \ldots \mathrm{M}-2)$

Figure 2-3. The paths of the relative rapidities in the case $\pi / 2<\mu<2 \pi / 3$.

are determined from this behavior of the rapidities. They are rearranged as

$$
\begin{aligned}
& \lambda_{M}^{(f)}=\lambda_{1}^{(i)}, \quad \lambda_{M-1}^{(f)}=\lambda_{2}^{(i)}, \quad(j=1, \cdots, M-2) . \\
& \lambda_{j}^{(f)}=\lambda_{j+2}^{(i)}
\end{aligned}
$$

Let us determine the relation between $\left\{p_{j}^{(i)}, \theta\left(p_{j}^{(i)}, p_{k}^{(i)}\right)\right\}$ and $\left\{p_{j}^{(f)}, \theta\left(p_{j}^{(f)}, p_{k}^{(f)}\right)\right\}$. 
The cut of the function $p(\lambda)$ extends from the branch point $i \mu$ to another branch point $i(2 \pi-\mu)$. The path of $\lambda_{1}$ starts from the branch point $i(2 \pi-\mu)$ and that of $\lambda_{M}$ reaches the same branch point $i(2 \pi-\mu)$ (Fig. 2-2). To determine the relation between $p_{M}^{(f)}$ and $p_{1}^{(i)}$, we follow a circuit consisting of the path of $\lambda_{M}$, that of $\lambda_{1}$ and the real axis. We start from the branch point $\lambda_{1}^{(i)}=i(2 \pi-\mu)$ perpendicularly to the imaginary axis. Finally we come to the same branch point $\lambda_{M}^{(f)}=i(2 \pi-\mu)$ perpendicularly to the imaginary axis after running through the circuit. The circuit encircles the 'half' of the branch point $\lambda=i(2 \pi-\mu)$ of the function $p(\lambda)$. We conclude that $p\left(\lambda_{M}^{(f)}\right)$ is larger than $p\left(\lambda_{1}^{(i)}\right)$ by $\pi$ :

$$
p_{M}^{(f)}=p_{1}^{(i)}+\pi
$$

Similarly we find the relation

$$
p_{M-1}^{(f)}=p_{2}^{(i)}+\pi
$$

The other relations are trivial

$$
p_{j}^{(f)}=p_{j+2}^{(f)} \quad(j=1, \ldots, M-2) .
$$

The cut of the function $\theta(\lambda)$ extends from the branch point $2 i(\pi-\mu)$ to another branch point $2 i \mu$. To determine the relation between $\theta\left(p_{M}^{(f)}, p_{M-1}^{(f)}\right)$ and $\theta\left(p_{1}^{(i)}, p_{2}^{(i)}\right)$ we consider the circuit consisting of the paths of the relative rapidities $\lambda_{M}-\lambda_{M-1}$, $\lambda_{1}-\lambda_{2}$ and the real axis. This circuit crosses this cut (Fig. 2-3). Therefore the relation between the two phase shifts is

$$
\theta\left(p_{M}^{(f)}, p_{M-1}^{(f)}\right)=\theta\left(p_{1}^{(i)}, p_{2}^{(i)}\right)+2 \pi
$$

To consider the other relations for the phase shifts, recall that the difference in the two rapidities in the two-string is larger than $2(\pi-\mu)$ in this process. From this we say the relation $\operatorname{Im}\left(\lambda_{M}\right) \geq \pi+(\pi-\mu)=2 \pi-\mu$ and $\operatorname{Im}\left(\lambda_{M-1}\right) \leq \pi-(\pi-\mu)=\mu$ 
in the two-string. Therefore $\operatorname{Im}\left(\lambda_{M}-\lambda_{j}\right) \geq 2 \pi-\mu>2 \mu(j \neq M, M-1)$ and $\operatorname{Im}\left(\lambda_{M-1}-\lambda_{j}\right) \leq \mu<2(\pi-\mu)(j \neq M, M-1)$ are satisfied in the region of our consideration $\frac{\pi}{2}<\mu<\frac{3}{2} \pi$. This means that the circuit consisting of the paths of $\lambda_{M}-\lambda_{j}$, that of $\lambda_{1}-\lambda_{j+2}$ and the real axis does not cross the cut of the phase shift function (Fig. 2-3). Similarly the circuit consisting of the paths of $\lambda_{M-1}-\lambda_{j}$, $\lambda_{2}-\lambda_{j+2}$ and the real axis does not cross the cut (Fig. 2-3). From these results we obtain the relations

$$
\begin{aligned}
\theta\left(p_{M}^{(f)}, p_{j}^{(f)}\right) & =\theta\left(p_{1}^{(i)}, p_{j+2}^{(i)}\right), \\
\theta\left(p_{M-1}^{(f)}, p_{j}^{(f)}\right) & =\theta\left(p_{2}^{(i)}, p_{j+2}^{(i)}\right),
\end{aligned}
$$

where $j=1, \ldots, M-2$. The other relations are trivial

$$
\theta\left(p_{j}^{(f)}, p_{k}^{(f)}\right)=\theta\left(p_{j+2}^{(i)}, p_{k+2}^{(i)}\right)
$$

where $j, k=1, \ldots, M-2$.

The set $\left\{p_{j}^{(i)}\right\}$ and the set $\left\{p_{j}^{(f)}\right\}$ are not equivalent even up to $2 \pi$, as seen in (2.8) and (2.9). In spite of this, the initial state $(\Phi=-2 \pi)$ and the final state $(\Phi=2 \pi)$ are found to be the same. We show this in Appendix B.

Our conclusion (2.8), (2.9) and (2.10) are different from the one derived in [19]. In ref. [19], it was concluded that the two-string never comes close enough to the $i \pi$ line. They have obtained the relations $p_{M}^{(f)}=p_{1}^{(i)}, p_{M-1}^{(f)}=p_{2}^{(i)}$ and $p_{j}^{(f)}=p_{j+2}^{(i)}$. Their conclusion is not valid however. By summing over the relations (1.5) for all $j(j=1, \ldots, M)$, we obtain a simple relation

$$
\sum_{j=1}^{M} p_{j}=\frac{\Phi}{2},
$$

which must be satisfied. Their conclusion does not satisfy this simple consistency condition. 
Finally, let us check briefly the consistency between the boundary conditions (1.5)and our relations (2.8)-(2.13). The boundary conditions for the final state are

$$
2 M p_{j}^{(f)}+\sum_{k \neq j} \theta\left(p_{j}^{(f)}, p_{k}^{(f)}\right)=2 \pi\left(\frac{2 j-M-1}{2}\right)+2 \pi, \quad(j=1, \ldots, M)
$$

These equations are rewritten in terms of the initial set by using (2.8)-(2.13).

$$
\begin{array}{r}
2 M\left(p_{M}^{i}+\pi\right)+\left(\sum_{k \neq 1} \theta\left(p_{1}^{(i)}, p_{k}^{(i)}\right)+2 \pi\right)=2 \pi\left(\frac{M-1}{2}\right)+2 \pi, \\
2 M\left(p_{2}^{(i)}+\pi\right)+\left(\sum_{k \neq 2} \theta\left(p_{2}^{(i)}, p_{k}^{(i)}\right)-2 \pi\right)=2 \pi\left(\frac{M-3}{2}\right)+2 \pi, \\
2 M p_{j+2}^{(i)}+\sum_{k \neq j+2} \theta\left(p_{j+2}^{(i)}, p_{k}^{(i)}\right)=2 \pi\left(\frac{2 j-M-1}{2}\right)+2 \pi, \\
(j=1, \ldots, M-2) .
\end{array}
$$

They are equivalent to the boundary conditions for the initial state

$$
\begin{gathered}
2 M p_{j+2}^{(i)}+\sum_{k \neq j+2} \theta\left(p_{j+2}^{(i)}, p_{k}^{(i)}\right)=2 \pi\left(\frac{2(j+2)-M-1}{2}\right)-2 \pi, \\
(j=-1,0, \ldots, M-2) .
\end{gathered}
$$

\subsection{Ground state in the Case of three String}

Let us now turn to the case of three-string which is formed in the region $\cos (\pi / 3)<\Delta<\cos (\pi / 4),\left(\frac{2}{3} \pi<\mu<\frac{3}{4} \pi\right)$. We first describe the behavior of the rapidities we have found in this region. When $\Phi$ increases from zero to $2(\pi-\mu)$, the largest rapidity $\lambda_{M}$ goes to infinity and jumps onto the $i \pi$ line. When $\Phi$ increases from $2(\pi-\mu)$ to $4(\pi-\mu)$, the second largest rapidity $\lambda_{M-1}$ 
goes to infinity and the two rapidities jump by $i \pi / 2$ simultaneously. Thus the two rapidities $\lambda_{M}=\infty+\frac{3}{2} i \pi$ and $\lambda_{M-1}=\infty+\frac{1}{2} i \pi$ form a two-string as seen in $\S 2.1$. As $\Phi$ increases further from $4(\pi-\mu)$, the two-string starts moving from plus infinity. The center of the two-string is on the $i \pi$ line and the distance between $\lambda_{M}$ and $\lambda_{M-1}$ becomes shorter. The two-string and the third largest rapidity $\lambda_{M-2}$ attract each other. When $\Phi=6(\pi-\mu)$, the two-string and $\lambda_{M-2}$ go to infinity. Here the distance between $\lambda_{M}$ and $\lambda_{M-1}$ becomes $2 \pi / 3$. Three rapidities are, therefore, $\lambda_{M}=\infty+\frac{4}{3} i \pi, \quad \lambda_{M-1}=\infty+\frac{2}{3} i \pi, \quad \lambda_{M-2}=\infty$. Here the three rapidities jump by $\frac{\pi}{3} i$. They form a three-string where $\lambda_{M}=\infty+\frac{5}{3} i \pi, \lambda_{M-1}=\infty+i \pi$ and $\lambda_{M-2}=\infty+\frac{1}{3} i \pi$. The deviation of the length of the string due to the finite size in $(1.7)$ is $\delta_{M} \equiv \delta_{1}^{(3)}=2 i\left(\frac{2}{3} \pi-\mu\right), \delta_{M-1} \equiv \delta_{2}^{(3)}=0$ and $\delta_{M-2} \equiv \delta_{3}^{(3)}=-2 i\left(\frac{2}{3} \pi-\mu\right)$.

When $\Phi$ increases from $6(\pi-\mu)$ to $4 \pi-6(\pi-\mu)$, the center of the three-string moves from $\infty+i \pi$ to $-\infty+i \pi$. The three-string fluctuates in this process (Fig. $2-4)$. It is interesting to note that the deviation $\delta_{M}\left(\delta_{M-2}\right)$ moves counterclockwise (clockwise) $M-1$ times around the origin $(\delta=0)$ which is the singular point where the phase shift diverges. This singular point in the relative rapidity space is the branch point of the phase shift function $\theta(\lambda)$. Thus how many times the relative rapidity winds around the branch point determines the corresponding increment of the phase shift. When the relative rapidity $\lambda$ moves counterclockwise around the branch point $\lambda=2 i(\pi-\mu)$, the phase shift increases by $2 \pi$. The phase shift $\theta\left(\lambda_{M}, \lambda_{M-1}\right)\left(\theta\left(\lambda_{M-1}, \lambda_{M-2}\right)\right)$ increases (decreases) by $2(M-1) \pi$ due to the fluctuation of the three string.

At $\Phi=4 \pi-6(\pi-\mu)$, the center of the three-string reaches $-\infty+i \pi$. The rapidities jump by $-\frac{\pi}{3}$ and are decomposed into $\lambda_{M-2}$ on the real axis and the two-string $\left(\lambda_{M}-\lambda_{M-1}\right)$ whose center is on $i \pi$ line. At $\Phi=4 \pi-4(\pi-\mu)$ the two-string is decomposed; $\lambda_{M}$ and $\lambda_{M-1}$ jump onto the $i \pi$ line and the real axis respectively. Finally $\lambda_{M}$ jumps on to the real axis at $\Phi=4 \pi-2(\pi-\mu)$. When $\Phi=4 \pi$, the set of the rapidities $\left\{\lambda_{j}^{(f)}\right\}$ is the same as the set of initial $(\Phi=0)$ 


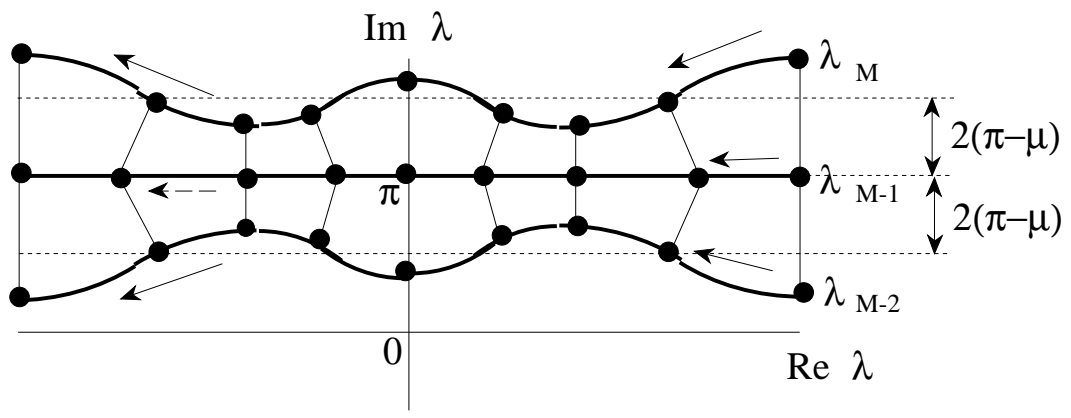

Figure 2-4. The fluctuations of the three-string in the case of $M=3$ and $\mathrm{N}=6$.

rapidities $\left\{\lambda_{j}^{(i)}\right\}$. The rapidities are rearranged as

$$
\begin{aligned}
& \lambda_{M}^{(f)}=\lambda_{1}^{(i)}, \quad \lambda_{M-1}^{(f)}=\lambda_{2}^{(i)}, \quad \lambda_{M-2}^{(f)}=\lambda_{3}^{(i)}, \\
& \lambda_{j}^{(f)}=\lambda_{j+3}^{(i)}(j=1, \ldots, M-3) .
\end{aligned}
$$

This behavior of rapidities has been checked by numerical calculation (Fig. 25). The motion of the two-string is independent of the rapidities lying on the real axis as seen in $\S 2-1$. In the case of the three-string, its fluctuation is influenced by the rapidities lying on the real axis.

The relations between the set $\left\{p_{j}^{(f)}, \theta\left(p_{j}^{(f)}, p_{k}^{(f)}\right)\right\}$ and the set $\left\{p_{j}^{(i)}, \theta\left(p_{j}^{(i)}, p_{k}^{(i)}\right)\right\}$ are determined from the behavior of the rapidities. The branch cut of the function 


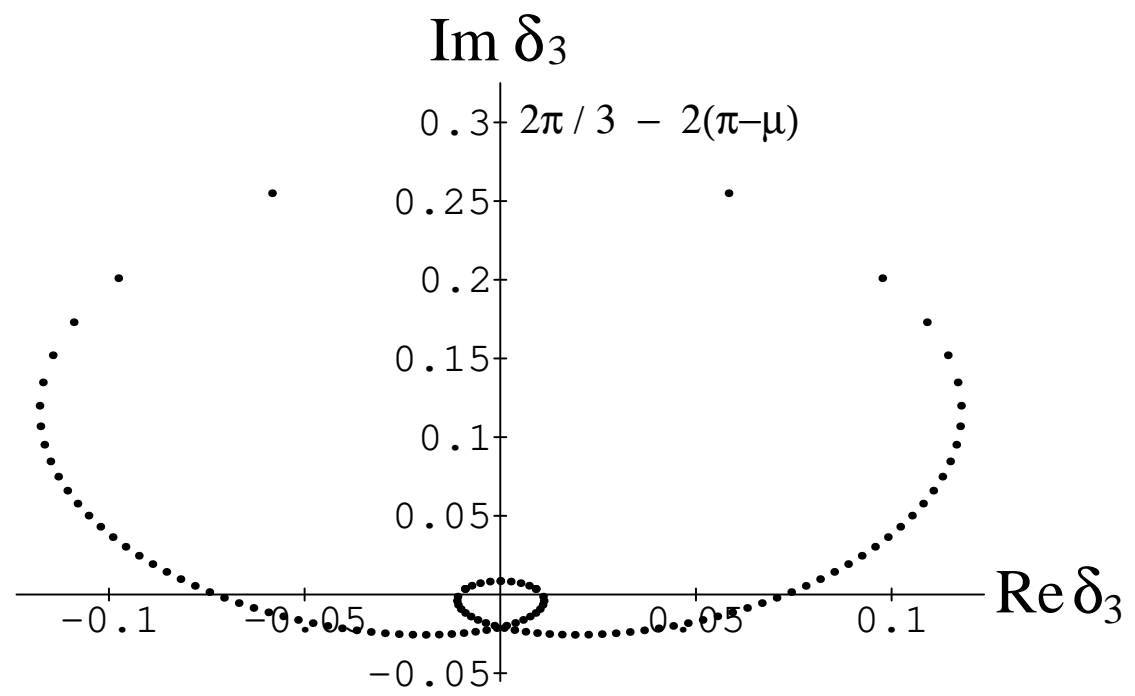

Figure 2-5. The deviation $\delta_{3}=\left(\lambda_{3}-\lambda_{2}\right)-2(\pi-\mu)$ in the case of $M=3, N=6$ and $\mu=5 \pi / 7$. When $\Phi=6(\pi-\mu)$, the deviation is $2 \pi / 3-2(\pi-\mu) \simeq 0.299$. When $\Phi$ increases from $6(\pi-\mu)$ to $4 \pi-6(\pi-\mu)$, the deviation moves counterclockwise twice around the origin.

$p(\lambda)$ extends from the branch point $\lambda=i \mu$ to another branch point $\lambda=i(2 \pi-\mu)$. Only the path of $\lambda_{M-1}$ crosses the cut (Fig. 2-6), so that the relations of momenta are

$$
\begin{aligned}
& p_{M}^{(f)}=p_{1}^{(i)}, \quad p_{M-1}^{(f)}=p_{2}^{(i)}+2 \pi, \quad p_{M-2}^{(f)}=p_{3}^{(i)}, \\
& p_{j}^{(f)}=p_{j+3}^{(i)}(j=1, \ldots, M-3) .
\end{aligned}
$$

The cut of $\theta(\lambda)$ extends from the branch point $\lambda=2 i(\pi-\mu)$ to another branch point $\lambda=2 i \mu$. The path of the relative rapidity $\lambda_{M}-\lambda_{M-1}$, and those of $\lambda_{M-1}-\lambda_{M-2}$ and $\lambda_{M}-\lambda_{M-2}$ cross the cut $1+(M-1)$ times, $1-(M-1)$ times and once respectively (Fig. 2-7). We have, therefore,

$$
\begin{aligned}
\theta\left(\lambda_{M}^{(f)}, \lambda_{M-1}^{(f)}\right) & =\theta\left(\lambda_{1}^{(i)}, \lambda_{2}^{(i)}\right)+2 M \pi \\
\theta\left(\lambda_{M-1}^{(f)}, \lambda_{M-2}^{(f)}\right) & =\theta\left(\lambda_{2}^{(i)}, \lambda_{3}^{(i)}\right)-2(M-2) \pi, \\
\theta\left(\lambda_{M}^{(f)}, \lambda_{M-2}^{(f)}\right) & =\theta\left(\lambda_{1}^{(i)}, \lambda_{3}^{(i)}\right)+2 \pi .
\end{aligned}
$$




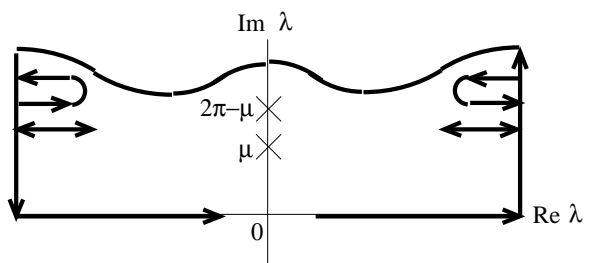

(a) the path of $\lambda_{\mathrm{M}}$

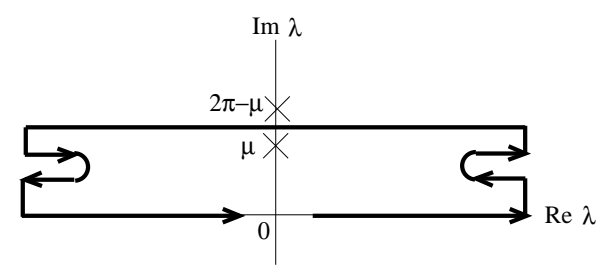

(b) the path of $\lambda_{\mathrm{M}-1}$

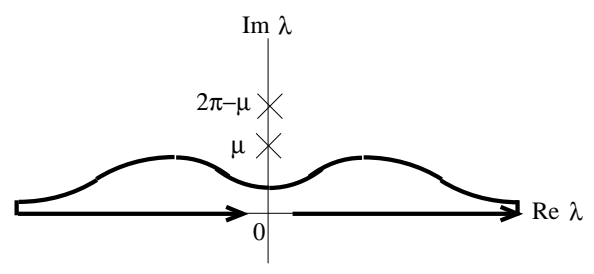

(c) the path of $\lambda_{\mathrm{M}-2}$

Figure 2-6. The paths of the rapidities in the case of $2 \pi / 3<\mu<3 \pi / 4$.

For the other phase shifts, only the path $\lambda_{M-1}-\lambda_{k}(k=1, \ldots, M-3)$ crosses the cut once (Fig. 2-8). We have

$$
\begin{aligned}
\theta\left(\lambda_{M}^{(f)}, \lambda_{k}^{(f)}\right) & =\theta\left(\lambda_{1}^{(i)}, \lambda_{k+3}^{(i)}\right), \\
\theta\left(\lambda_{M-1}^{(f)}, \lambda_{k}^{(f)}\right) & =\theta\left(\lambda_{2}^{(i)}, \lambda_{k+3}^{(i)}\right)+2 \pi, \\
\theta\left(\lambda_{M-2}^{(f)}, \lambda_{k}^{(f)}\right) & =\theta\left(\lambda_{3}^{(i)}, \lambda_{k+3}^{(i)}\right), \\
\theta\left(\lambda_{j}^{(f)}, \lambda_{k}^{(f)}\right) & =\theta\left(\lambda_{j+3}^{(i)}, \lambda_{k+3}^{(i)}\right), \\
& (j, k=1, \ldots, M-3) .
\end{aligned}
$$

Finally, let us check the consistency between the relations (2.21)-(2.23) and the twisted boundary conditions (1.5). The boundary conditions for the final state 


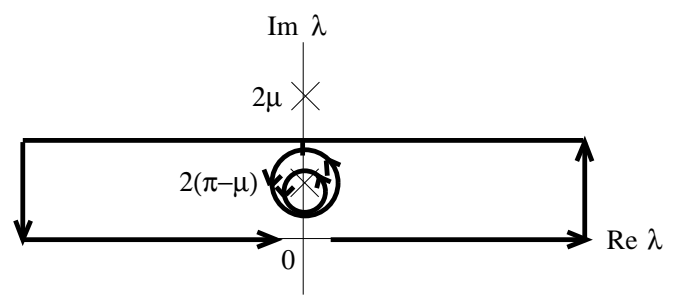

(a) the path of $\left(\lambda_{M}-\lambda_{M-1}\right)$

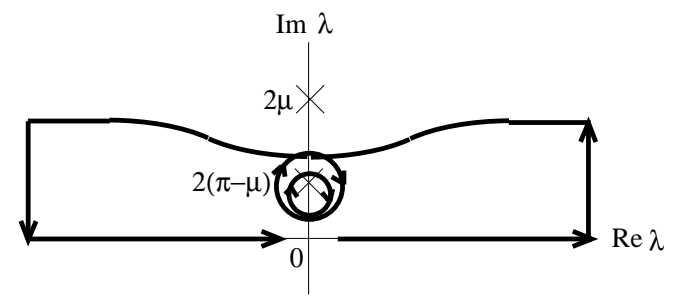

(b) the path of $\left(\lambda_{\mathrm{M}-1}-\lambda_{\mathrm{M}-2}\right)$

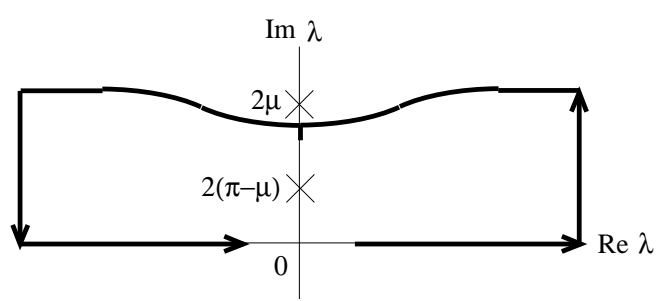

(c) the path of $\left(\lambda_{M}-\lambda_{M-2}\right)$

Figure 2-7. The paths of the relative rapidities in the case of $2 \pi / 3<\mu<$ $3 \pi / 4$.

are

$$
\begin{gathered}
2 M p_{j}^{(f)}+\sum_{k \neq j} \theta\left(p_{j}^{(f)}, p_{k}^{(f)}\right)=2 \pi\left(\frac{2 j-M-1}{2}\right)+4 \pi \\
(j=1, \ldots, M) .
\end{gathered}
$$

These equations are rewritten in terms of the initial set by using (2.21)-(2.23),

$$
\begin{gathered}
2 M p_{1}^{(i)}+\left(\sum_{k \neq 1} \theta\left(p_{1}^{(i)}, p_{j+3}^{(i)}\right)+2 M \pi+2 \pi\right)=2 \pi\left(\frac{M-1}{2}\right)+4 \pi \\
2 M\left(p_{2}^{(i)}+2 \pi\right)+\left(\sum_{k \neq 2} \theta\left(p_{2}^{(i)}, p_{j+3}^{(i)}\right)-2 M \pi-2(M-2) \pi+2(M-3) \pi\right)=2 \pi\left(\frac{M-3}{2}\right)+4 \pi
\end{gathered}
$$




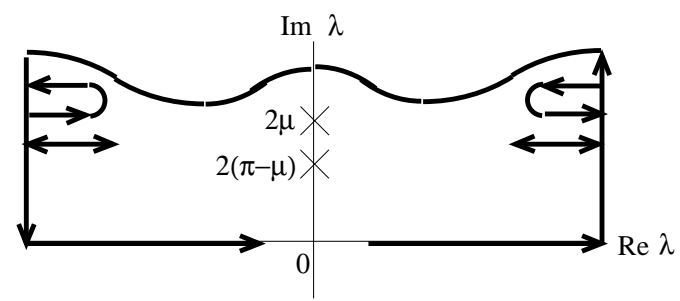

(a) the path of $\left(\lambda_{M}-\lambda_{j}\right)$

$$
(\mathrm{j}=1,2, \ldots, \mathrm{M}-3)
$$

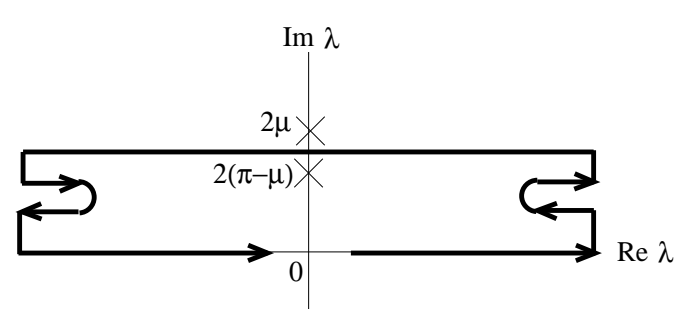

(b) the path of $\left(\lambda_{\mathrm{M}-1}-\lambda_{\mathrm{j}}\right)$ $(\mathrm{j}=1,2, \ldots, \mathrm{M}-3)$

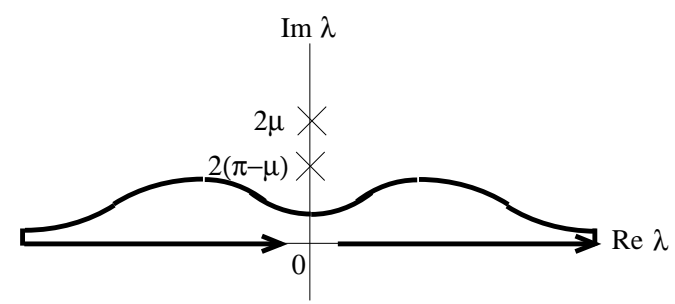

(c) the path of $\left(\lambda_{\mathrm{M}-2}-\lambda_{\mathrm{j}}\right)$ $(\mathrm{j}=1,2, \ldots, \mathrm{M}-3)$

Figure 2-8. The paths of the relative rapidities in the case of $2 \pi / 3<\mu<$ $3 \pi / 4$.

$$
2 M p_{3}^{(i)}+\left(\sum_{k \neq 3} \theta\left(p_{3}^{(i)}, p_{j+3}^{(i)}\right)+2(M-2) \pi-2 \pi\right)=2 \pi\left(\frac{M-5}{2}\right)+4 \pi
$$

$2 M p_{j+3}^{(i)}+\left(\sum_{k \neq j+3} \theta\left(p_{j+3}^{(i)}, p_{j+3}^{(i)}\right)-2 \pi\right)=2 \pi\left(\frac{2 j-M-1}{2}\right)+4 \pi, \quad(j=1, \ldots, M-3)$ 
They are equivalent to the boundary conditions for the initial state:

$$
\begin{gathered}
2 M p_{1}+\sum_{k \neq 1} \theta\left(p_{1}^{(i)}, p_{k}^{(i)}\right)=2 \pi\left(-\frac{M-1}{2}\right)+0, \\
2 M p_{2}+\sum_{k \neq 2} \theta\left(p_{2}^{(i)}, p_{k}^{(i)}\right)=2 \pi\left(-\frac{M-3}{2}\right)+0, \\
2 M p_{3}+\sum_{k \neq 3} \theta\left(p_{3}^{(i)}, p_{k}^{(i)}\right)=2 \pi\left(-\frac{M-5}{2}\right)+0, \\
2 M p_{j+3}+\sum_{k \neq j+3} \theta\left(p_{j+3}^{(i)}, p_{k}^{(i)}\right)=2 \pi\left(-\frac{2 j+3-M-1}{2}\right)+0, \\
(j=1, \ldots, M-3) .
\end{gathered}
$$

\subsection{Generalization to The CASE OF $n$-STRing}

We have found the collision of the rapidities forming the two-string into the singularities as well as the fluctuation of the three-string around its center in the previous subsections. It is possible to generalize these behaviors to the case of $n$-string. Let us describe the motion of the rapidities we have found in the region $\cos \left(\frac{\pi}{n}\right)<\Delta<\cos \left(\frac{\pi}{n+1}\right)\left(\frac{n-1}{n} \pi<\mu<\frac{n}{n+1} \pi\right)$. At $\Phi=0$, all rapidities are symmetrically arranged on the real axis with respect to the origin. At $\Phi=2 j(\pi-\mu)$ for $(j=1,2, \cdots, n)$, a group of $j$ rapidities at $\operatorname{Re}(\lambda)=\infty$ jumps: a $j$-string is formed from a $(j-1)$-string and one string. The outcome of this series of events is the formation of the $n$-string at $\Phi=2 n(\pi-\mu)$. After this, the center of the $n$-string moves backwards on the $i \pi$ line from $\infty+i \pi$ to $-\infty+i \pi$ as $\Phi$ increases from $2 n(\pi-\mu)$ to $4 \pi-2(\pi-\mu)$. The $n$-string itself fluctuates during this period as we have seen in the case of the three-string. (We will describe this shortly for the case $n$ even and for the case $n$ odd separately.) As $\Phi$ increases further, a separation of a $\mathrm{j}$-string into a ( $\mathrm{j}-1)$-string and one string on the real axis occurs at $\Phi=4 \pi-2 j(\pi-\mu)$ for $j=n, n-1, \cdots, 1$. Finally at $\Phi=4 \pi$, all rapidities are again symmetrically arranged on the real axis with respect to the origin. 
Let us now describe the fluctuation of the $n$-string. In the case of $n$ even, the two rapidities in the middle, namely, $\lambda_{M-\frac{n}{2}+1}$ and $\lambda_{M-\frac{n}{2}}$ of the $n$-string collide into the two branch points of $p(\lambda)$ at $\Phi=2 \pi$ :

$$
\lambda_{M-\frac{n}{2}+1}=2 \pi-\mu, \quad \lambda_{M-\frac{n}{2}}=\mu .
$$

The other rapidities fluctuate: the deviation

$$
\delta_{M-j+1} \equiv\left(\lambda_{M-j+1}-\lambda_{M-j}\right)-2(\pi-\mu) \quad\left(j=1,2, \cdots, \frac{n}{2}-1\right)
$$

moves counterclockwise $(M-n+2 j)$ times around $\delta=0$, which is the branch point of $\theta(\lambda)$. The deviation

$$
\delta_{M-n+j} \equiv\left(\lambda_{M-n+j}-\lambda_{M-n+j+1}\right)-2(\pi-\mu) \quad\left(j=1,2, \cdots, \frac{n}{2}-1\right)
$$

moves clockwise $(M-n+2 j)$ times around $\delta=0$.

In the case of $n$ odd, the rapidity in the middle $\lambda_{M-\frac{n-1}{2}}$ moves on the $i \pi$ line while the other rapidities fluctuate: the deviation

$$
\delta_{M-j+1} \equiv\left(\lambda_{M-j+1}-\lambda_{M-j}\right)-2(\pi-\mu) \quad\left(j=1,2, \cdots, \frac{n-1}{2}\right)
$$

moves counterclockwise $(M-n+2 j)$ times around $\delta=0$ and the deviation

$$
\delta_{M-n+j} \equiv\left(\lambda_{M-n+j}-\lambda_{M-n+j+1}\right)-2(\pi-\mu) \quad\left(j=1,2, \cdots, \frac{n-1}{2}\right)
$$

moves clockwise $(M-n+2 j)$ times around $\delta=0$. Only the rapidity $\lambda_{M-\frac{n-1}{2}}$ crosses the cut.

The rationale for this behavior of the rapidities lies in the fact that they pass a set of consistency conditions, which is found to be very stringent. Let us denote by 
$\left\{\lambda_{j}^{(i)}\right\}$ a set of initial rapidities at $\Phi=-2 \pi$ and by $\left\{\lambda_{j}^{(f)}\right\}$ a set of final rapidities at $\Phi=2 \pi$. We have

$$
\begin{aligned}
\lambda_{M-j+1}^{(f)} & =\lambda_{j}^{(i)} \quad(j=1,2, \cdots, n), \\
\lambda_{j}^{(f)} & =\lambda_{j+n}^{(i)}(j=1,2, \cdots, M-n)
\end{aligned} .
$$

From the paths of the rapidities described above, we obtain a set of relations for the momenta

$$
\begin{gathered}
p\left(\lambda_{M-j+1}^{(f)}\right)=p\left(\lambda_{j}^{(i)}\right), \quad p\left(\lambda_{M-n+j}^{(f)}\right)=p\left(\lambda_{n-j+1}^{(i)}\right) \quad\left(j=1,2, \cdots,\left[\frac{n-1}{2}\right]\right) . \\
p\left(\lambda_{M-\frac{n}{2}+1}^{(f)}\right)=p\left(\lambda_{\frac{n}{2}}^{(i)}\right)+\pi, \quad p\left(\lambda_{M-\frac{n}{2}}^{(f)}\right)=p\left(\lambda_{\frac{n}{2}+1}^{(i)}\right)+\pi \quad(n: \text { even }) . \\
p\left(\lambda_{M-\frac{n-1}{2}}^{(f)}\right)=P\left(\lambda_{\frac{n+1}{2}}^{(i)}\right)+2 \pi \quad(n: \text { odd }) . \\
p\left(\lambda_{j}^{(f)}\right)=p\left(\lambda_{j+n}^{(i)}\right) \quad(j=1,2, \cdots, M-n) .
\end{gathered}
$$

From the paths of the relative rapidities, we obtain a set of relations for the phase shifts:

$$
\begin{gathered}
\theta\left(\lambda_{M-j+1}^{(f)}, \lambda_{M-j}^{(f)}\right)=\theta\left(\lambda_{j}^{(i)}, \lambda_{j+1}^{(i)}\right)+2 \pi\{1+(M-n+2 j)\} \\
\left(j=1,2, \cdots,\left[\frac{n-1}{2}\right]\right) . \\
\theta\left(\lambda_{M-n+j}^{(f)}, \lambda_{M-n+j+1}^{(f)}\right)=\theta\left(\lambda_{n-j+1}^{(i)}, \lambda_{j}^{(i)}\right)+2 \pi\{1+(M-n+2 j)\} \\
\left(j=1,2, \cdots,\left[\frac{n-1}{2}\right]\right) . \\
\theta\left(\lambda_{M-\frac{n}{2}+1}^{(f)}, \lambda_{M-\frac{n}{2}}^{(f)}\right)=\theta\left(\lambda_{\frac{n}{2}-1}^{(i)}, \lambda_{\frac{n}{2}}^{(i)}\right)+2 \pi, \\
\theta\left(\lambda_{M-j+1}^{(f)}, \lambda_{M-k+1}^{(f)}\right)=\theta\left(\lambda_{j}^{(i)}, \lambda_{k}^{(i)}\right)+2 \pi \quad(1 \leq j<k \leq n \text { and } k-j \geq 2),
\end{gathered}
$$




$$
\begin{gathered}
\theta\left(\lambda_{M}^{(f)}, \lambda_{k}^{(f)}\right)=\theta\left(\lambda_{1}^{(i)}, \lambda_{k+n}^{(i)}\right) \quad(k=1, \cdots, M-n), \\
\theta\left(\lambda_{M-n+1}^{(f)}, \lambda_{k}^{(f)}\right)=\theta\left(\lambda_{n}^{(i)}, \lambda_{k+n}^{(i)}\right) \quad(k=1, \cdots, M-n), \\
\theta\left(\lambda_{M-j+1}^{(f)}, \lambda_{k}^{(f)}\right)=\theta\left(\lambda_{j}^{(i)}, \lambda_{k+n}^{(i)}\right)+2 \pi \quad(2 \leq j \leq n-1, k=1, \cdots, M-n), \\
\theta\left(\lambda_{j}^{(f)}, \lambda_{k}^{(f)}\right)=\theta\left(\lambda_{j+n}^{(i)}, \lambda_{k+n}^{(i)}\right) \quad(j=1, \cdots, M-n, k=1, \cdots, M-n) .
\end{gathered}
$$

In the first and the second formulas, the factor $\pm(M-n+2 j)$ represents a contribution due to the fluctuation of the $n$-string. The third formula is for the case of $n$ even only. Following the same procedure as described in $\S 2.1$ and $§ 2.2$, and using the relations given above, we have checked that the twisted boundary conditions for the set of the initial rapidities $\left\{\lambda_{j}^{(i)}\right\}$ follow from those for the set of the final rapidities $\left\{\lambda_{j}^{(f)}\right\}$. The proof is too long and tedious to be presented here.

This consistency check is in fact very stringent. We are convinced that the picture of the $n$-string fluctuations we have found in this paper has now been established by this. 


\section{Results in the Regime $\Delta \leq-1$}

In this section, we study the ground state properties mentioned in the introduction of the XXZ model in the regime $\Delta \leq-1$. Throughout this regime, the single particle energy is kept non-positive.

\subsection{THE REGIME $\Delta<-1$}

In this regime, the momentum is parametrized in terms of the rapidity as

$$
p(\lambda)=-i \ln \left[-\frac{\sinh \frac{1}{2}(i \lambda+\mu)}{\sinh \frac{1}{2}(i \lambda-\mu)}\right],
$$

where $\Delta=-\cosh \mu$. As the momentum increases from zero to $\pi$ (from $\pi$ to $2 \pi$ ), the rapidity increases from zero to $\pi$ (from $\pi$ to $2 \pi$ ). The particle-particle phase shift becomes

$$
\theta\left(\lambda_{1}, \lambda_{2}\right)=i \ln \left[-\frac{\sinh \frac{1}{2}\left(i\left(\lambda_{1}-\lambda_{2}\right)+2 \mu\right)}{\sinh \frac{1}{2}\left(i\left(\lambda_{1}-\lambda_{2}\right)-2 \mu\right)}\right]
$$

As the relative rapidity increases from zero to $\pi$ (from $\pi$ to $2 \pi$ ), the phase shift decreases from zero to $-\pi$ (from $-\pi$ to $-2 \pi$ ). We plot the momentum $p(\lambda)$ as a function of the rapidity and the phase shift $\theta(\lambda)$ as a function of the relative rapidity $\lambda$ in Fig. 3-1. The total energy is expressed as

$$
E=\frac{N}{2} \cosh \mu+\sum_{j=1}^{M} \frac{-2 \sinh ^{2} \mu}{\cosh \mu-\cos \lambda_{j}} .
$$

The single-particle energy

$$
\varepsilon_{j}=\frac{-2 \sinh ^{2} \mu}{\cosh \mu-\cos \lambda_{j}}
$$

is negative for the real $\lambda_{j}$. 


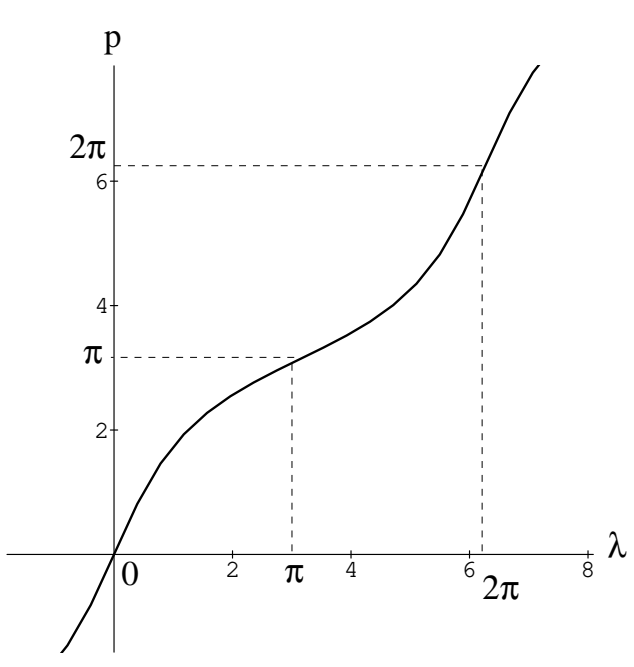

(a)

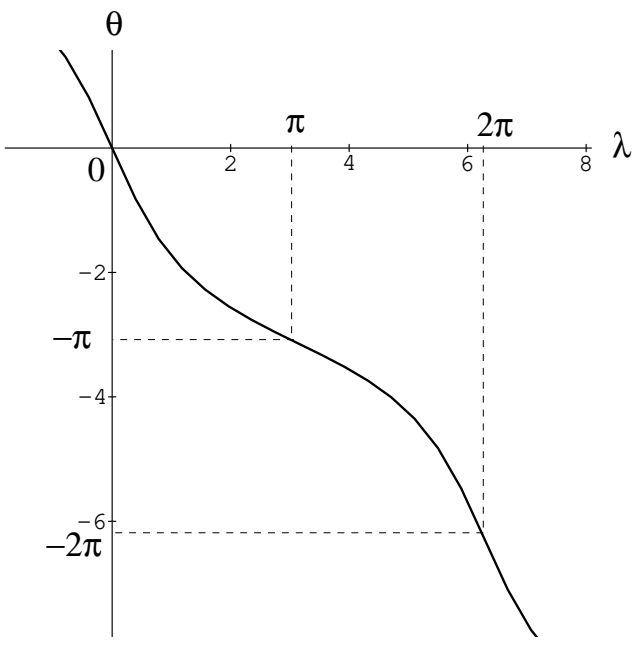

(b)

Figure 3-1. The function for the momentum and the function for the phase-shift function in the case of $\Delta<-1$.

Let us consider the process associated with the change of the twist angle $\Phi$ from zero to $4 \pi$. The motion of the rapidities is determined from equation (1.5) with (2.5). When $\Phi=0$, all the rapidities $\lambda_{j}$ are real and symmetric with respect to the origin of the rapidity space $\left(\left\{\lambda_{j}\right\}=\left\{-\lambda_{j}\right\}\right)$ within the Brillouin zone $-\pi<\lambda<\pi$. At $\Phi=2 \pi$, the largest rapidity $\lambda_{M}$ (or one-string) reaches $\pi$ and the rest of them are symmetric with respect to the origin of the rapidity plane $\lambda=0$. This is an excited state for the periodic boundary condition. At $\Phi=4 \pi$ we will denote the configuration of $\lambda_{j}$ by $\left\{\lambda_{j}^{(f)}\right\}$. It is related to the original ground state configuration by

$$
\lambda_{M}^{(f)}=\lambda_{1}^{(i)}+2 \pi, \quad \lambda_{j}^{(f)}=\lambda_{j+1}^{(i)} \quad(j \neq M) .
$$

This leads to the relations

$$
p_{M}^{(f)}=p_{1}^{(i)}+2 \pi, \quad p_{j}^{(f)}=p_{j+1}^{(i)} \quad(j \neq M),
$$




$$
\begin{gathered}
\theta\left(p_{M}^{(f)}, p_{j}^{(f)}\right)=\theta\left(p_{1}^{(i)}, p_{j+1}^{(i)}\right)-2 \pi, \\
\theta\left(p_{j}^{(f)}, p_{k}^{(f)}\right)=\theta\left(p_{j+1}^{(i)}, p_{k+1}^{(i)}\right) \quad(j \neq M, k \neq M),
\end{gathered}
$$

where $p_{j}^{(f)}=p\left(\lambda_{j}^{(f)}\right)$ and $p_{j}^{(i)}=p\left(\lambda_{j}^{(i)}\right)$. The set of the momenta for the final state coincides with the one for the initial state up to $2 \pi$. Accordingly the system returns to the ground state at $\Phi=4 \pi$.

We check briefly the consistency between the above equation and the twisted boundary condition (1.5) at $\Phi=0,2 \pi, 4 \pi$. The twisted boundary condition for $\Phi=0$ is

$$
2 M p\left(\lambda_{j}^{(i)}\right)+\sum_{k \neq j} \theta\left(\lambda_{j}^{(i)}, \lambda_{k}^{(i)}\right)=2 \pi \frac{2 j-M-1}{2} .
$$

As the $p(\lambda)$ and $\theta(\lambda)$ are odd functions, the above equation becomes

$$
2 M p\left(-\lambda_{j}^{(i)}\right)+\sum_{k \neq j} \theta\left(-\lambda_{j}^{(i)},-\lambda_{k}^{(i)}\right)=2 \pi \frac{-M+2(M-j+1)-1}{2} .
$$

Comparing this with the twisted boundary condition for $p\left(\lambda_{M-j+1}^{i}\right)$

$$
2 M p\left(\lambda_{M-j+1}^{(i)}\right)+\sum_{k \neq j} \theta\left(\lambda_{M-j+1}^{i}, \lambda_{M-k+1}^{i}\right)=2 \pi \frac{-M+2(M-j+1)-1}{2},
$$

we find that the configuration of the rapidities is symmetric $\lambda_{M-j+1}^{(i)}=-\lambda_{j}^{(i)}$. In the case of $\Phi=2 \pi$, we can derive $\lambda_{M}=\pi$ and $\lambda_{M-j}=-\lambda_{j}(j \neq M)$ in the same way. When $\Phi=4 \pi$, the twisted boundary condition for $p\left(\lambda_{j}^{(f)}\right)$ is

$$
2 M p\left(\lambda_{j}^{(f)}\right)+\sum_{k \neq j} \theta\left(\lambda_{j}^{(f)}, \lambda_{k}^{(f)}\right)=2 \pi \frac{2 j-M-1}{2}+4 \pi
$$

Using Eqs. (3.6) and (3.7), the left hand side of the equation is rewritten in terms 
of $\lambda_{j}^{(i)}$,

$$
2 M p\left(\lambda_{j+1}^{(i)}\right)+\left(\sum_{k \neq j+1} \theta\left(\lambda_{j+1}^{(i)}, \lambda_{k}^{(i)}\right)+2 \pi\right)=2 \pi \frac{2 j-M-1}{2}+4 \pi,
$$

for $j \neq M$ and

$$
2 M p\left(\lambda_{1}^{(i)}\right)+\sum_{k \neq 1}\left(\theta\left(\lambda_{1}^{(i)}, \lambda_{k}^{(i)}\right)-2 \pi\right)=2 \pi \frac{M-1}{2}+4 \pi
$$

where we used the relation $\theta\left(\lambda_{j}, \lambda_{k}\right)=-\theta\left(\lambda_{k}, \lambda_{j}\right)$. Eqs. (3.12) and (3.13) are equivalent to the boundary conditions for the initial state

$$
\begin{gathered}
2 M p\left(\lambda_{j+1}^{(i)}\right)+\sum_{k \neq j+1} \theta\left(\lambda_{j+1}^{(i)}, \lambda_{k}^{(i)}\right)=2 \pi \frac{2(j+1)-M-1}{2}+0, \\
2 M p\left(\lambda_{1}^{(i)}\right)+\sum_{k \neq 1} \theta\left(\lambda_{1}^{(i)}, \lambda_{k}^{(i)}\right)=2 \pi\left(-\frac{M-1}{2}\right)+0 .
\end{gathered}
$$

We confirm the motion of the rapidities for the case of $M=2$ and $N=4$ in Appendix A. Observe that the single-particle energy (3.4) remains negative during the process.

\subsection{THE $\Delta=-1$ CASE}

Let us now study the ground state in the critical coupling case $\Delta=-1$. When $\Delta=-1$, the momentum $p$ is parametrized in terms of rapidity $\lambda$ by

$$
p(\lambda)=2 \arctan (2 \lambda)
$$

When the momentum increases from zero to $\pi$, the rapidity goes from zero to $+\infty$. As the momentum increases still more the rapidity jumps from $+\infty$ to $-\infty$. When 
the momentum increase from $\pi$ to $2 \pi$, the rapidity goes from $-\infty$ to zero. The phase shift and total energy become

$$
\theta\left(\lambda_{1}, \lambda_{2}\right)=-2 \arctan \left(\lambda_{1}-\lambda_{2}\right)
$$

and

$$
E=\frac{N}{2}+\sum_{j=1}^{M} \frac{-1}{1+4 \lambda_{j}^{2}}
$$

respectively. The single-particle energy

$$
\varepsilon_{j}=\frac{-1}{1+4 \lambda_{j}^{2}}
$$

is negative for real $\lambda_{j}$ and zero for $\lambda_{j}= \pm \infty$.

Let us describe the process associated with the change of twist angle $\Phi$. When $\Phi=0$, all the rapidities $\lambda_{j}$ are real and symmetric with respect to the origin of the rapidity space. If we set $\Phi=2 \pi$, the largest rapidity $\lambda_{M}$ goes to plus infinity and jumps to minus infinity. At $\Phi=4 \pi$ the configuration of rapidities $\left\{\lambda_{j}\right\}$ comes back to the initial configuration as follows

$$
\lambda_{M}^{(f)}=\lambda_{1}^{(i)}, \quad \lambda_{j}^{(f)}=\lambda_{j+1}^{(i)} \quad(j \neq M) .
$$

This leads to the relations

$$
\begin{aligned}
p_{M}^{(f)}= & p_{1}^{(i)}+2 \pi, \quad p_{j}^{(f)}=p_{j+1}^{(i)} \quad(j \neq M), \\
& \theta\left(p_{M}^{(f)}, p_{j}^{(f)}\right)=\theta\left(p_{1}^{(i)}, p_{j+1}^{(i)}\right)-2 \pi, \\
& \theta\left(p_{j}^{(f)}, p_{k}^{(f)}\right)=\theta\left(p_{j+1}^{(i)}, p_{k+1}^{(i)}\right) .
\end{aligned}
$$

This is the same as the relations (3.6) and (3.7) in the $\Delta<-1$ case. The period of the system is again $\Delta \Phi=4 \pi$. 
We have found that the motion of the rapidities, the period, the final configuration of the momenta and that of the phase shift functions in the case $\Delta \leq-1$ are the same as those found in the case $-1<\Delta<0$. We have, therefore, established the continuity of the two regimes. This can be heuristically explained by the structure of the degeneracies of the levels, which we illustrate for the case $M=2, N=4$ in Fig. $3-2$. The ground state at $\Phi=0$ never gets degenerated with the zero energy levels as long as $\Delta<0$. This is, however, seen for $\Delta \geq 0$.

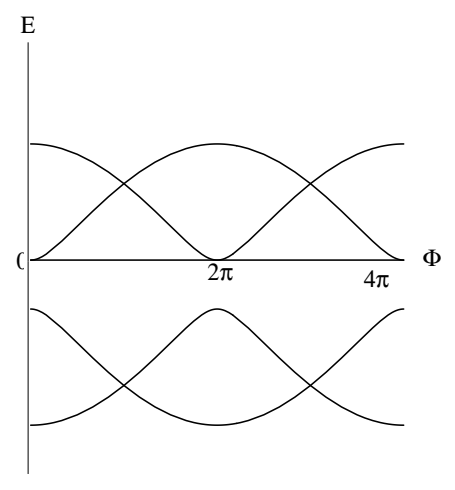

(a) $\Delta<0$

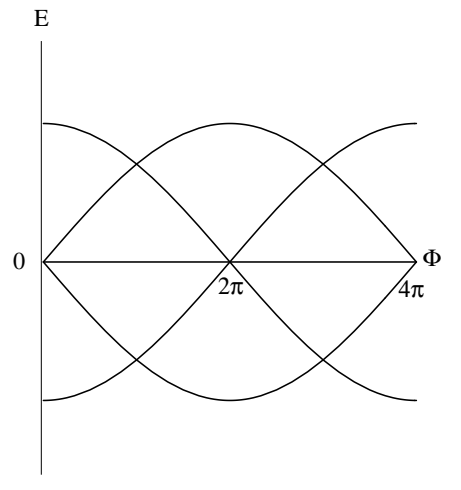

(b) $\Delta=0$

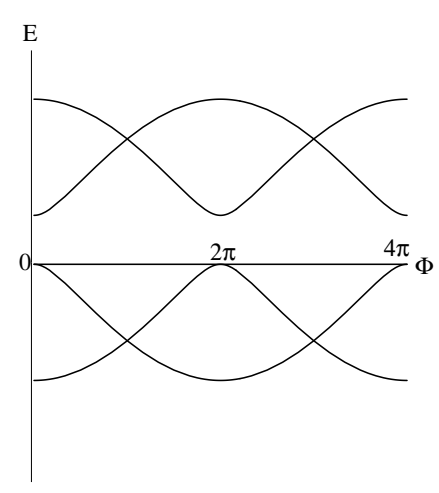

(c) $\Delta>0$

Figure 3-2. The energy levels of XXZ model in $\mathrm{M}=2$ and $\mathrm{N}=4$ case. The eigenenergies are $E=\Delta \pm \sqrt{\Delta^{2}+8 \cos ^{2} \Phi / 4}, \Delta \pm \sqrt{\Delta^{2}+8 \sin ^{2} \Phi / 4}, 0,0$ which we derive in Appendix A. 


\section{The Berry Phase}

Let us now turn to the discussion of the Berry phase associated with the twist angle $\Phi(t)$, which is now considered to vary with time. We are interested in computing the nontrivial geometrical phase $\gamma$ appearing in the wave function

$$
|\psi(\Phi(t), t)\rangle=\exp \left\{-i \int_{0}^{t} E_{0}(\Phi(t)) d t\right\} \exp (i \gamma(t))\left|\psi_{0}(\Phi(t))\right\rangle .
$$

The substitution of this into the Schrodinger equation provides a formula

$$
\gamma(t)=\operatorname{Re}\left[i \int_{0}^{t} d t \frac{\left\langle\psi_{0}(\Phi(t))\left|\frac{\partial}{\partial \Phi}\right| \psi_{0}(\Phi(t))\right\rangle}{\left\langle\psi_{0}(\Phi(t)) \mid \psi_{0}(\Phi(t))\right\rangle} \frac{d \Phi}{d t}\right] .
$$

In our consideration, the initial and the final twist angles are taken to be $\Phi(0)=$ $-2 \pi$ and $\Phi(t)=+2 \pi$ respectively. After a change of variables, we find

$$
\gamma=\operatorname{Re}\left[i \int_{-2 \pi}^{2 \pi} d \Phi \frac{\left\langle\psi_{0}(\Phi)\left|\frac{\partial}{\partial \Phi}\right| \psi_{0}(\Phi)\right\rangle}{\left\langle\psi_{0}(\Phi) \mid \psi_{0}(\Phi)\right\rangle}\right]
$$

We compute this quantity in different regions.

\section{1. $\Delta \leq-1$}

We take the half-integer coordinate $x_{j}=-\frac{N-1}{2},-\frac{N-3}{2}, \ldots, \frac{N-1}{2}$, where $N=$ $2 M$. We first examine the relation between the wave function $\chi(\Phi)$ of the initial state at $\Phi=-2 \pi$ and that of the final state at $\Phi=2 \pi$. We first want to establish $($ see $(1.2))$

$$
|\chi(-2 \pi)\rangle=-|\chi(2 \pi)\rangle
$$

By using the permutation

$$
Q_{a}^{\prime}=\left\{\begin{array}{rc}
Q_{a}+1 & \left(\text { for } Q_{a} \neq M\right), \\
1 & \left(\text { for } Q_{a}=M\right),
\end{array}\right.
$$

and the relations (3.5), (3.6) and (3.7) we find the difference in the phase factors 
from the initial state to the final state :

$$
\begin{aligned}
(-1)^{|Q|} & =(-1)^{\left|Q^{\prime}\right|} \times(-1)^{M-1} \\
\exp \left(i \sum_{a=1}^{M} x_{a} p_{Q_{a}}^{(f)}\right) & =\exp \left(i \sum_{a=1}^{M} x_{a} p_{Q_{a}^{\prime}}^{(i)}\right) \times(-1) \\
\exp \left(\frac{i}{2} \sum_{a<b} \theta\left(p_{Q_{b}}^{(i)}, p_{Q_{a}}^{(i)}\right) \epsilon\left(x_{b}-x_{a}\right)\right) & =\exp \left(\frac{i}{2} \sum_{a<b} \theta\left(p_{Q_{b}^{\prime}}^{(f)}, p_{Q_{a}^{\prime}}^{(f)}\right) \epsilon\left(x_{b}-x_{a}\right)\right) \times(-1)^{M-1}
\end{aligned}
$$

Thus under the full period $\Phi=4 \pi, \chi$ gets multiplied by a factor $(-1)^{M-1}(-1)(-1)^{M-1}=$ -1 , which is what we wanted to show.

Identifying

$$
\left|\psi_{0}(\Phi)\right\rangle=\exp (-i \Phi / 4)|\chi(\Phi)\rangle
$$

We find

$$
\gamma=\pi+\operatorname{Re}\left[i \int_{-2 \pi}^{2 \pi} d \Phi \sum_{\left\{x_{i}\right\}} \frac{\chi\left(x_{1}, \ldots, x_{M}\right)^{*} \frac{\partial}{\partial \Phi} \chi\left(x_{1}, \ldots, x_{M}\right)}{\chi\left(x_{1}, \ldots, x_{M}\right)^{*} \chi\left(x_{1}, \ldots, x_{M}\right)}\right] .
$$

The summation $\sum_{\left\{x_{i}\right\}}$ represents $\prod_{i=1}^{M} \sum_{x_{i}}$, where each $x_{i}$ runs over the region of half integer values. The function of $\Phi$

$$
\sum_{\left\{x_{i}\right\}} \frac{\chi\left(x_{1}, \ldots, x_{M} \mid p_{1}(\Phi), \ldots p_{M}(\Phi)\right)^{*} \frac{\partial}{\partial \Phi} \chi\left(x_{1}, \ldots, x_{M} \mid p_{1}(\Phi), \ldots p_{M}(\Phi)\right)}{\|\chi(\Phi)\|^{2}}
$$

is odd as shown in Appendix B. The second term in (4.10) is zero and the Berry phase is equal to

$$
\gamma=\pi
$$

Recalling the calculation of ref [18], we find that the Berry phase is $\pi$ throughout the region $\Delta<0$. This confirms the continuity at $\Delta=-1$. It will be different in the region $\Delta>0$, which we will find in the next subsections. 


\section{2. $0<\Delta<\cos \pi / 3$}

The strategy for the computation is the same. By using the permutation

$$
Q_{a}^{\prime}=\left\{\begin{aligned}
Q_{a}+2, & \left(\text { for } Q_{a} \neq M-1, M\right), \\
2, & \left(\text { for } Q_{a}=M-1\right), \\
1, & \left(\text { for } Q_{a}=M\right),
\end{aligned}\right.
$$

the relations $(2.8)-(2.13)$ are expressed as

$$
\begin{gathered}
p_{Q_{a}}^{(f)}=p_{Q_{a}^{\prime}}^{(i)}+\pi\left(\delta_{Q_{a}^{\prime}, 1}+\delta_{Q_{a}^{\prime}, 2}\right), \\
\theta\left(p_{Q_{b}}^{(f)}, p_{Q_{a}}^{(f)}\right)=\theta\left(p_{Q_{b}^{\prime}}^{(i)}, p_{Q_{a}^{\prime}}^{(i)}\right)+2 \pi \delta_{Q_{b}^{\prime}, 1} \delta_{Q_{a}^{\prime}, 2}-2 \pi \delta_{Q_{b}^{\prime}, 2} \delta_{Q_{a}^{\prime}, 1} .
\end{gathered}
$$

Counting the change of the phase factors

$$
\begin{gathered}
(-1)^{|Q|}=(-1)^{\left|Q^{\prime}\right|} \times(-1), \\
\exp \left[i \sum_{a=1}^{M} x_{a} p_{Q_{a}}^{(f)}\right]=\exp \left[i \sum_{a=1}^{M} x_{a} p_{Q_{a}^{\prime}}^{(i)}\right], \\
\exp \left[\frac{i}{2} \sum_{a<b} \theta\left(p_{Q_{b}}^{(f)}, p_{Q_{a}}^{(f)}\right) \epsilon\left(x_{b}-x_{a}\right)\right]=\exp \left[\frac{i}{2} \sum_{a<b} \theta\left(p_{Q_{b}^{\prime}}^{(i)}, p_{Q_{a}^{\prime}}^{(i)}\right) \epsilon\left(x_{b}-x_{a}\right)\right] \times(-1),
\end{gathered}
$$

we find, in the same way as we have derived Eqs. (4.4),

$$
|\chi(-2 \pi)\rangle=|\chi(2 \pi)\rangle
$$

We conclude

$$
\gamma=0
$$




\section{3. $\cos \pi / 3<\Delta<\cos \pi / 4$}

The corresponding formulas in this case are

$$
\begin{gathered}
Q_{a}^{\prime}=\left\{\begin{array}{cc}
Q_{3}+3, & \left(\text { for } Q_{a} \neq M-2, M-1, M\right), \\
3, & \left(\text { for } Q_{a}=M-2\right), \\
2, & \left(\text { for } Q_{a}=M-1\right), \\
1, & \left(\text { for } Q_{a}=M\right), \\
\theta\left(p_{Q_{b}}^{(f)}, p_{Q_{a}}^{(f)}\right)=\theta\left(p_{Q_{b}^{\prime}}^{(i)}, p_{Q_{a}^{\prime}}^{(i)}\right)+2 M \pi\left(\delta_{Q_{b}^{\prime}, 1} \delta_{Q_{a}^{\prime}, 2}-\delta_{Q_{b}^{\prime}, 2} \delta_{Q_{a}^{\prime}, 1}\right) \\
+2(M-2) \pi\left(\delta_{Q_{b}^{\prime}, 2} \delta_{Q_{a}^{\prime}, 3}-\delta_{Q_{b}^{\prime}, 3} \delta_{Q_{a}^{\prime}, 2}\right) \\
+2 \pi\left(\delta_{Q_{b}^{\prime}, 1} \delta_{Q_{a}^{\prime}, 3}-\delta_{Q_{b}^{\prime}, 3} \delta_{Q_{a}^{\prime}, 1}\right) \\
+2 \pi\left\{\delta_{Q_{b}^{\prime}, 2} \sum_{k=4}^{M} \delta_{Q_{a}^{\prime}, k}-\sum_{k=4}^{(i)} \delta_{Q_{b}^{\prime}, k} \delta_{Q_{a}^{\prime}, 2}\right.
\end{array}\right\}
\end{gathered}
$$

and

$$
\begin{gathered}
(-1)^{|Q|}=(-1)^{\left|Q^{\prime}\right|} \times(-1)^{(M-1)+(M-2)+(M-3)} \\
\exp \left[i \sum_{a=1}^{M} x_{a} p_{Q_{a}}^{(f)}\right]=\exp \left[i \sum_{a=1}^{M} x_{a} p_{Q_{a}^{\prime}}^{(i)}\right] \times(-1), \\
\mathrm{e}^{\frac{i}{2} \sum_{a<b} \theta\left(p_{Q_{b}}^{(f)}, p_{Q_{a}}^{(f)}\right) \epsilon\left(x_{b}-x_{a}\right)}=\mathrm{e}^{\frac{i}{2} \sum_{a<b} \theta\left(p_{Q_{b}^{\prime}}^{(i)}, p_{Q_{a}^{\prime}}^{(i)}\right) \epsilon\left(x_{b}-x_{a}\right)} \times(-1)^{M+(M-2)+1+(M-3)} .
\end{gathered}
$$

We conclude

$$
\gamma=\pi
$$




\section{4. $\cos (\pi / n) \leq \Delta \leq \cos (\pi / n+1)$}

By using the relations we have found in $\S 2.3$., we compute the Bery phase in this regime in the same way as we did in $n=2, n=3$. The final answer is

$$
\begin{aligned}
& \gamma=0 \quad \text { for } n \text { even } \\
& \gamma=\pi \quad \text { for } n \text { odd. }
\end{aligned}
$$

\subsection{Discussion}

Finally we would like to argue that the Berry phase we have computed can be used as a measure of the statistics of the quasiparticle ( or the bound state) involved in the process. The above calculation shows

$$
\gamma / \pi=m \quad \bmod 2 .
$$

for the case in which the $m$-string goes around the loop in the momentum/rapidity plane. This quantity $\gamma / \pi \bmod 2$ measures the monodromy property in the $p$ space of the particle involved. We propose to use this as a definition of statistics. To make our discussion a little more concrete, let us consider the case $\Delta=-1$. At $\Phi=2 \pi$, the largest rapidity ( or one-string) goes to the edge of the Brillouin zone

( or infinity in the $\lambda$ plane.) This root ( or the rapidity) decouples from the rest of the roots and the system of $M$ equations eq. (1.5)reduces that of $M-1$ equations. According to the description of [21], this is a two-spinon state over the vacuum. Eq. (4.26)provides 1 for a creation and a subsequent annihilation of two spinons, which implies $1 / 2$ for a spinon. 


\section{Acknowledgements}

We thank Vladimir Korepin for valuable discussion on this subject. We are also grateful to Yasuhiro Akutsu, Kiyoshi Higashijima, Keiji Kikkawa and V. P. Nair for insightful remarks.

\section{Note Added}

After the completion of this paper, we became aware that the formation and the collision of the two-string roots as a function of the coupling and the twist angle is mentioned by Alcaraz, Barber and Batchelor in [22]. We thank Professor Batchelor for informing us of this reference. 


\section{APPENDIX A}

In this appendix we examine the case $M=2, N=4$. The boundary conditions (1.5) are

$$
\begin{aligned}
& 4 p_{1}-\theta\left(p_{2}, p_{1}\right)=-\pi+\Phi \\
& 4 p_{2}-\theta\left(p_{2}, p_{1}\right)=+\pi+\Phi
\end{aligned}
$$

while the phase shift

$$
\theta\left(p_{2}, p_{1}\right)=2 \arctan \left[\frac{\Delta \sin \left[\left(p_{2}-p_{1}\right) / 2\right]}{\cos \left[\left(p_{2}+p_{1}\right) / 2\right]-\Delta \cos \left[\left(p_{2}-p_{1}\right) / 2\right]}\right]
$$

In what follows, we solve explicitly this system of equations. The sum (A.1) +(A.2) leads to the relation

$$
p_{2}+p_{1}=\frac{\Phi}{2}
$$

So the phase shift (A.3) becomes

$$
\theta\left(p_{2}, p_{1}\right)=2 \arctan \left[\frac{\Delta \sin \left[\left(p_{2}-p_{1}\right) / 2\right]}{\cos [\Phi / 4]-\Delta \cos \left[\left(p_{2}-p_{1}\right) / 2\right]}\right]
$$

From difference (A.2) - (A.1) we obtain another formula for the phase-shift.

$$
\theta\left(p_{2}, p_{1}\right)=\pi-2\left(p_{2}-p_{1}\right)
$$

These formulas of the phase shift (A.5) and (A.6) lead to the equation on the relative momentum

$$
\arctan \left[\frac{\Delta \sin q}{\cos (\Phi / 4)-\Delta \cos q}\right]=\pi-4 q
$$

where $q \equiv\left(p_{2}-p_{1}\right) / 2$. This is expressed as a relation

$$
2 \cos \frac{\Phi}{4} \cos ^{2} q-\Delta \cos q-\cos \frac{\Phi}{4}=0
$$


When $\cos (\Phi / 4)=0$, the equation becomes

$$
\cos q=0
$$

When $\cos (\Phi / 4) \neq 0$, the roots of the equation (A.7) are

$$
\cos q=\frac{\Delta \pm \sqrt{\Delta^{2}+8 \cos ^{2}(\Phi / 4)}}{4 \cos (\Phi / 4)}
$$

In the case $|\cos q| \leq 1, p_{1}$ and $p_{2}$ are real number. In the case of $|\cos q|>1, p_{1}$ and $p_{2}$ are complex number with $p_{1}{ }^{*}=p_{2}$. This set of the complex momenta is the two-string. When $\cos (\Phi / 4)$ goes to zero, the relative momentum becomes

$$
\begin{aligned}
& \lim _{\cos (\Phi / 4) \rightarrow+0} \cos q^{(+)} \equiv \lim _{\cos (\Phi / 4) \rightarrow 0} \frac{\Delta+\sqrt{\Delta^{2}+8 \cos ^{2}(\Phi / 4)}}{4 \cos (\Phi / 4)}=\left\{\begin{array}{rr}
+\infty & \text { for } \Delta>0, \\
0 & \text { for } \Delta<0,
\end{array}\right. \\
& \lim _{\cos (\Phi / 4) \rightarrow+0} \cos q^{(-)} \equiv \lim _{\cos (\Phi / 4) \rightarrow 0} \frac{\Delta-\sqrt{\Delta^{2}+8 \cos ^{2}(\Phi / 4)}}{4 \cos (\Phi / 4)} \\
&=\left\{\begin{array}{rr}
0 & \text { for } \Delta>0, \\
+\infty & \text { for } \Delta<0 .
\end{array}\right.
\end{aligned}
$$

From (A.4) and (A.8), $p_{1}$ and $p_{2}$ are determined as

$$
\begin{aligned}
& p_{1}=\frac{\Phi}{4}-\arccos \left[\frac{\Delta \pm \sqrt{\Delta^{2}+8 \cos ^{2}(\Phi / 4)}}{4 \cos (\Phi / 4)}\right] \\
& p_{2}=\frac{\Phi}{4}+\arccos \left[\frac{\Delta \pm \sqrt{\Delta^{2}+8 \cos ^{2}(\Phi / 4)}}{4 \cos (\Phi / 4)}\right]
\end{aligned}
$$

The wave function is determined from the momenta as follows. In the present 
system the wave function (1.2) with (1.3) is

$$
\begin{aligned}
|\chi\rangle= & \sum_{x_{1}} \sum_{x_{2}} \epsilon\left(x_{2}-x_{1}\right) \sum_{Q}(-1)^{|Q|} \exp \left(i x_{1} p_{Q_{1}}+i x_{2} p_{Q_{2}}\right) \\
& \times \exp \left(\frac{i}{2} \theta\left(p_{Q_{2}}, p_{Q_{1}}\right) \epsilon\left(x_{2}-x_{1}\right)\right) \sigma_{x_{1}}^{-} \sigma_{x_{2}}^{-}|\uparrow\rangle
\end{aligned}
$$

The wave function is rewritten as

$$
\begin{aligned}
|\chi\rangle= & \sum_{\left\{x_{1}, x_{2} \mid x_{2}>x_{1}\right\}}\left\{\exp \left(i x_{1} p_{1}+i x_{2} p_{2}+\frac{i}{2} \theta\left(p_{1}, p_{2}\right)\right)\right. \\
& \left.-\exp \left(i x_{1} p_{2}+i x_{2} p_{1}-\frac{i}{2} \theta\left(p_{1}, p_{2}\right)\right)\right\} \sigma_{x_{1}}^{-} \sigma_{x_{2}}^{-}|\uparrow\rangle \\
= & 4 i \sum_{\left\{x_{1}, x_{2} \mid x_{2}>x_{1}\right\}} \exp \left(i \frac{p_{1}+p_{2}}{2}\left(x_{2}+x_{1}\right)\right) \\
& \times \sin \left(i \frac{p_{2}-p_{1}}{2}\left(x_{2}-x_{1}\right)+\frac{1}{2} \theta\left(p_{1}, p_{2}\right)\right) \sigma_{x_{1}}^{-} \sigma_{x_{2}}^{-}|\uparrow\rangle
\end{aligned}
$$

By using (A.4) and (A.6), the wave function becomes

$$
|\chi\rangle=4 i \sum_{\left\{x_{1}, x_{2} \mid x_{2}>x_{1}\right\}} \exp \left(i \frac{\Phi}{4}\left(x_{2}+x_{1}\right)\right) \cos \left(\frac{p_{2}-p_{1}}{2}\left(x_{2}-x_{1}-2\right)\right) \sigma_{x_{1}}^{-} \sigma_{x_{2}}^{-}|\uparrow\rangle .
$$

We take the coordinate as

$$
\{x\}=\left\{-\frac{3}{2}, \frac{1}{2}, \frac{1}{2}, \frac{3}{2} \cdot\right\}
$$

The wave function is described in terms of the relative momentum;

$$
\begin{aligned}
|\chi\rangle= & 4 i] \exp \left(-i \frac{\Phi}{4}\right)\left|-\frac{3}{2}, \frac{1}{2}\right\rangle+\exp \left(+i \frac{\Phi}{4}\right)\left|-\frac{1}{2}, \frac{3}{2}\right\rangle \\
& \left.+i \cos \frac{p_{2}-p_{1}}{2}\left\{\exp \left(-i \frac{\Phi}{4}\right)\left|-\frac{3}{2},-\frac{1}{2}\right\rangle+\left|-\frac{1}{2}, \frac{1}{2}\right\rangle+\exp \left(+i \frac{\Phi}{4}\right)\left|\frac{1}{2}, \frac{3}{2}\right\rangle+\left|\frac{3}{2},-\frac{3}{2}\right\rangle\right\}\right]
\end{aligned}
$$

We get the explicit formula of the wave function by substituting (A.8) into this. 
The eigenenergy is calculated from (A.4) and (A.8)

$$
E=\Delta \mp \sqrt{\Delta^{2}+8 \cos ^{2}(\Phi / 4)} .
$$

The energy, relative momentum and the momenta of the ground state are

$$
\begin{gathered}
E^{(0)}=\Delta-\sqrt{\Delta^{2}+8 \cos ^{2}(\Phi / 4)} \\
\cos q^{(0)}=\frac{\Delta+\sqrt{\Delta^{2}+8 \cos ^{2}(\Phi / 4)}}{4 \cos \frac{\Phi}{4}}, \\
p_{1}^{(0)}=\frac{\Phi}{2}-\arccos \left[\frac{\Delta+\sqrt{\Delta^{2}+8 \cos ^{2}(\Phi / 4)}}{4 \cos \frac{\Phi}{4}}\right], \\
p_{2}^{(0)}=\frac{\Phi}{2}+\arccos \left[\frac{\Delta+\sqrt{\Delta^{2}+8 \cos ^{2}(\Phi / 4)}}{4 \cos \frac{\Phi}{4}}\right] .
\end{gathered}
$$

Let us consider the case $\Delta<0$. In this case the momentum is real number because of

$$
\left|\cos q^{(0)}\right|=\left|\frac{\Delta+\sqrt{\Delta^{2}+8 \cos ^{2}(\Phi / 4)}}{4 \cos \frac{\Phi}{4}}\right| \leq 1
$$

The motion of the momentum in the process is as follows. When $\Phi=0$, the momenta are

$$
\begin{aligned}
& p_{1}^{(i)}=-\arccos \left[\frac{\Delta+\sqrt{\Delta^{2}+8}}{4}\right], \\
& p_{2}^{(i)}=+\arccos \left[\frac{\Delta+\sqrt{\Delta^{2}+8}}{4}\right],
\end{aligned}
$$

thus the momentum is arranged symmetric $p_{1}^{(i)}=-p_{2}^{(i)}$. When $\Phi=2 \pi, \cos q^{(0)}=$ 0 , so the momenta are

$$
p_{1}=0, \quad p_{2}=\pi .
$$

When $\Phi=4 \pi$, the state comes back to the ground state. The set of momenta 
$\left\{p_{1}^{f}, p_{2}^{f}\right\}$ at $\Phi=4 \pi$ is equal to the set of momenta $\left\{p_{1}^{(i)}, p_{2}^{(i)}\right\}$ at $\Phi=0$ up to $2 \pi$;

$$
\begin{aligned}
p_{1}^{f} & =\frac{4 \pi}{4}-\arccos \left[-\frac{\Delta+\sqrt{\Delta^{2}+8}}{4}\right] \\
& =\pi-\left(\pi-\arccos \left[-\frac{\Delta+\sqrt{\Delta^{2}+8}}{4}\right]\right) \\
& =-\arccos \left[-\frac{\Delta+\sqrt{\Delta^{2}+8}}{4}\right] \\
& =p_{2}^{(i)}, \\
p_{2}^{f} & =\frac{4 \pi}{4}+\arccos \left[-\frac{\left.\Delta+\sqrt{\Delta^{2}+8}\right]}{4}\right] \\
& =\pi+\left(\pi-\arccos \left[-\frac{\Delta+\sqrt{\Delta^{2}+8}}{4}\right]\right) \\
& =2 \pi+p_{1}^{(i)},
\end{aligned}
$$

Our assertion on the motion of the rapidities in Sec.4-3 is checked by (A.10)-(A.11).

Next we consider the case $\Delta>0$. We take the region $-2 \pi<\Phi<2 \pi$ for convenience. Note the relation

$$
\begin{aligned}
\left|\cos q^{(0)}\right| & =\left|\frac{\Delta+\sqrt{\Delta^{2}+8 \cos ^{2}(\Phi / 4)}}{4 \cos \frac{\Phi}{4}}\right| \\
& \left\{\begin{array}{l}
\leq 1 \quad \text { for } \quad-2(\pi-\mu) \leq \Phi \leq 2(\pi-\mu), \\
>1 \quad \text { for } \quad-2 \pi<\Phi<-2(\pi-\mu), 2(\pi-\mu)<\Phi<2 \pi,
\end{array}\right.
\end{aligned}
$$

we check that the two-string is formed at $\Phi=2(\pi-\mu)$. In the two-string the momenta is complex number with $p_{1}^{*}=p_{2}$ which is equivalent to the center of rapidities $\left(\lambda_{1}, \lambda_{2}\right)$ is on the $i \pi$ line (see (2.1)). When $\Phi$ goes to $2 \pi$, the relative momentum become $q^{(0)} \rightarrow i \infty$ because of $\cos q^{(0)} \rightarrow \infty$, so the momentum becomes

$$
\begin{aligned}
p_{1}^{f} & =\frac{\pi}{2}-i \infty, \\
p_{2}^{f} & =\frac{\pi}{2}+i \infty .
\end{aligned}
$$


From this and Eq. (2.1), we find the rapidities arrived at the singular point

$$
\begin{aligned}
& \lambda_{1}^{f}=i(2 \pi-\mu), \\
& \lambda_{2}^{f}=i \mu .
\end{aligned}
$$

In the same way, we obtain the momenta and rapidities at $\Phi=-2 \pi$ as

$$
\begin{aligned}
p_{1}^{i} & =-\frac{\pi}{2}+i \infty \\
p_{2}^{i} & =-\frac{\pi}{2}-i \infty \\
\lambda_{1}^{i} & =i \mu \\
\lambda_{2}^{i} & =i(2 \pi-\mu) .
\end{aligned}
$$

So, we get the relations

$$
\begin{gathered}
\lambda_{2}^{f}=\lambda_{1}^{i}, \quad \lambda_{1}^{f}=\lambda_{2}^{i}, \\
p_{2}^{f}=p_{1}^{i}+\pi, \quad p_{1}^{f}=p_{2}^{i}+\pi .
\end{gathered}
$$

They agree with (2.7)-(2.8).

The set $\left\{p_{j}^{f}\right\}$ is not equivalent to the set $\left\{p_{j}^{i}\right\}$ up to $2 \pi$. However the initial state and final state are the same state. The relative momentum $\cos q^{(0)}=\infty$ at $\Phi= \pm 2 \pi$, thus the wave function (A.9) becomes

$$
\begin{aligned}
|\chi( \pm 2 \pi)\rangle & \propto\left\{\exp \left(-i \frac{ \pm 2 \pi}{4}\right)\left|-\frac{3}{2}, \frac{1}{2}\right\rangle+\exp \left(i \frac{ \pm 2 \pi}{4}\right)\left|-\frac{1}{2}, \frac{3}{2}\right\rangle\right\} \\
& +i^{2} \infty \times\left(\exp \left(-i \frac{ \pm 2 \pi}{2}\right)\left|-\frac{3}{2},-\frac{1}{2}\right\rangle+\left|-\frac{1}{2}, \frac{1}{2}\right\rangle+\exp \left(i \frac{ \pm 2 \pi}{2}\right)\left|\frac{1}{2}, \frac{3}{2}\right\rangle+\left|\frac{3}{2},-\frac{3}{2}\right\rangle\right)
\end{aligned}
$$

the normalized wave function is

$$
\begin{aligned}
|\chi( \pm 2 \pi)\rangle & =-\frac{1}{2}\left[\exp \left(-i \frac{ \pm 2 \pi}{2}\right)\left|-\frac{3}{2},-\frac{1}{2}\right\rangle+\left|-\frac{1}{2}, \frac{1}{2}\right\rangle+\exp \left(i \frac{ \pm 2 \pi}{2}\right)\left|\frac{1}{2}, \frac{3}{2}\right\rangle+\left|\frac{3}{2},-\frac{3}{2}\right\rangle\right] \\
& =-\frac{1}{2}\left[-\left|-\frac{3}{2},-\frac{1}{2}\right\rangle+\left|-\frac{1}{2}, \frac{1}{2}\right\rangle-\left|\frac{1}{2}, \frac{3}{2}\right\rangle+\left|\frac{3}{2},-\frac{3}{2}\right\rangle\right]
\end{aligned}
$$

Therefore we obtain $|\chi(2 \pi)\rangle=|\chi(-2 \pi)\rangle$. 
Finally we examine the energy spectrum by the explicit diagonalization of the Hamiltonian (1.6) with the twisted boundary condition which is equivalent to the Hamiltonian

$$
H=\sum_{j}\left(e^{i \frac{\Phi}{N}} \tilde{C}_{j}^{\dagger} \tilde{C}_{j+1}+e^{-i \frac{\Phi}{N}} \tilde{C}_{j+1}^{\dagger} \tilde{C}_{j}\right)-2 \Delta \sum_{j}\left(\tilde{C}_{j}^{\dagger} \tilde{C}_{j}-\frac{1}{2}\right)\left(\tilde{C}_{j+1}^{\dagger} \tilde{C}_{j+1}-\frac{1}{2}\right)
$$

with boundary condition

$$
\tilde{C}_{j+N}=\tilde{C}_{j}, \quad \tilde{C}_{j+N}^{\dagger}=\tilde{C}_{j}^{\dagger}
$$

where

$$
\tilde{C}_{j}=e^{i \frac{j}{N} \Phi} C_{j}, \quad \tilde{C}_{j}^{\dagger}=e^{-i \frac{j}{N} \Phi} C_{j}^{\dagger}
$$

The diagonalization of the Hamiltonian (A.12) for $N=4, M=2$ system leads to the energy spectrum

$$
E=\Delta \pm \sqrt{\Delta^{2}+8 \cos ^{2}\left(\frac{\Phi}{4}\right)}, \Delta \pm \sqrt{\Delta^{2}+8 \sin ^{2}\left(\frac{\Phi}{4}\right)}, 0,0
$$

We plot the spectrum as a function of $\Phi$ in the case $\Delta<0, \Delta=0$ and $\Delta>0$ in Sec. $4-4$. 


\section{APPENDIX B}

The set $\left\{p_{j}^{(i)}\right\}$ and the set $\left\{p_{j}^{(f)}\right\}$ are not equivalent even up to $2 \pi$, as seen in (2.8) and (2.9). In spite of this, the initial state $(\Phi=-2 \pi)$ and the final state $(\Phi=2 \pi)$ are the same. To show this, we see that the wave function $\mid \chi(-2 \pi)>$ of the final state (at $\Phi=2 \pi$ ) is invariant under the set of the transformations $p_{M}^{(f)} \rightarrow p_{M}^{(f)}+\pi$ and $p_{M-1}^{(f)} \rightarrow p_{M-1}^{(f)}+\pi$. When $\Phi=2 \pi-\epsilon(0<\epsilon \ll 1)$, the rapidities of two strings are $\lambda_{M}^{(f)} \simeq i(2 \pi-\mu)+\delta$ and $\lambda_{M-1}^{(f)} \simeq i \mu+\delta$, where $0<\delta \ll 1$. Substituting this into (2.1), we obtain

$$
p_{M}^{(f)} \simeq-i \Lambda+\frac{\pi}{2}, \quad p_{M-1}^{(f)} \simeq i \Lambda+\frac{\pi}{2}
$$

where $\Lambda \equiv \ln (2 \sin \mu / \delta) \gg 1 ; \Lambda$ goes to infinity for $\epsilon \rightarrow 0$. The phase shift $\theta\left(p_{M}^{(f)}, p_{M-1}^{(f)}\right)$ is determined from the twisted boundary conditions (see (1.5)),

$$
\begin{gathered}
2 M p_{M}^{(f)}+\theta\left(p_{M}^{(f)}, p_{M-1}^{(f)}\right)+\sum_{k=1}^{M-2} \theta\left(p_{M}^{(f)}, p_{k}^{(f)}\right)=2 \pi\left(\frac{M-1}{2}\right)+2 \pi, \\
2 M p_{M-1}^{(f)}+\theta\left(p_{M-1}^{(f)}, p_{M}^{(f)}\right)+\sum_{k=1}^{M-2} \theta\left(p_{M-1}^{(f)}, p_{k}^{(f)}\right)=2 \pi\left(\frac{M-3}{2}\right)+2 \pi .
\end{gathered}
$$

These lead to the relation

$$
\begin{aligned}
& 2 M\left(p_{M}^{(f)}-p_{M-1}^{(f)}\right)+2 \theta\left(p_{M}^{(f)}, p_{M-1}^{(f)}\right)+\sum_{k=1}^{M-2}\left(\theta\left(p_{M}^{(f)}, p_{k}^{(f)}\right)-\theta\left(p_{M-1}^{(f)}, p_{k}^{(f)}\right)\right) \\
= & 2 \pi .
\end{aligned}
$$

From (B.1) and (B.4) the phase shift becomes

$$
\theta\left(p_{M}^{(f)}, p_{M-1}^{(f)}\right) \simeq 2 i M \Lambda+\pi-\frac{1}{2} \sum_{k=1}^{M-2}\left(\theta\left(p_{M}^{(f)}, p_{k}^{(f)}\right)-\theta\left(p_{M-1}^{(f)}, p_{k}^{(f)}\right)\right)
$$

If we substitute this into the Bethe's wave function (1.2) and (1.3), the $\Lambda$ depen- 
dence of the wave function of the final state (at $\Phi=2 \pi$ ) becomes

$$
\begin{aligned}
& \quad|\chi(2 \pi)\rangle \\
& =\sum_{x_{1}, \cdots, x_{M}} \prod_{a<b} \epsilon\left(x_{b}-x_{a}\right) \sum_{Q}(-1)^{|Q|} \mathrm{e}^{i \sum_{a} x_{Q_{a}} p_{a}+\frac{i}{2} \sum_{a<b} \theta\left(p_{b}, p_{a}\right) \epsilon\left(x_{Q_{b}}-x_{Q_{a}}\right)} \prod_{j} \sigma_{x_{j}}^{-}|\uparrow\rangle \\
& =\sum_{x_{1}, \cdots, x_{M}} \prod_{a<b} \epsilon\left(x_{b}-x_{a}\right) \sum_{Q}(-1)^{|Q|} \exp \left[\Lambda\left(x_{Q_{M}}-x_{Q_{M-1}}-M \epsilon\left(x_{Q_{M}}-x_{Q_{M-1}}\right)\right)\right] \\
& \quad \times(\text { independent part of } \Lambda) \prod_{j} \sigma_{x_{j}}^{-}|\uparrow\rangle .
\end{aligned}
$$

Considering the range of the coordinate, it is shown that the leading terms $(\simeq$ $\left.e^{\Lambda(M-1)}\right)$ of the wave function (B.6) satisfy the condition $x_{Q_{M}}-x_{Q_{M-1}}=-1$ or $2 M-1:$

$$
\begin{aligned}
\left(x_{Q_{M}}, x_{Q_{M-1}}\right)= & \left(-\frac{N-1}{2},-\frac{N-3}{2}\right),\left(-\frac{N-3}{2},-\frac{N-5}{2}\right), \ldots \\
& \ldots,\left(\frac{N-5}{2}, \frac{N-3}{2}\right),\left(\frac{N-3}{2}, \frac{N-1}{2}\right),\left(\frac{N-1}{2},-\frac{N-1}{2}\right) .
\end{aligned}
$$

These $x_{Q_{M}}$ and $x_{Q_{M-1}}$ are adjacent to each other on the ring. When $\epsilon \rightarrow 0$ $(\Lambda \rightarrow \infty)$, the leading terms go to infinity faster than the other terms. Therefore, after the normalization of the wave function, the leading terms remain and the other terms vanish. The wave function at $\Phi=2 \pi(\epsilon \rightarrow 0)$ is the sum of the terms which satisfy the condition (B.7). Under the set of the transformations $p_{M}^{(f)} \rightarrow p_{M}^{(f)}+\pi$ and $p_{M-1}^{(f)} \rightarrow p_{M-1}^{(f)}+\pi$, the phase factor $\exp i \sum x_{Q_{a}} p_{a}$ in the wave function is transformed as

$$
\mathrm{e}^{i \sum x_{Q_{a}} p_{a}} \rightarrow \mathrm{e}^{i \sum x_{Q_{a}} p_{a}} \times \mathrm{e}^{i \pi\left(x_{Q_{M}}+x_{Q_{M-1}}\right)} .
$$

As $x_{Q_{M}}+x_{Q_{M-1}}$ is even as seen in (B.7), the phase factor in (B.8) becomes unity:

$$
\mathrm{e}^{i \pi\left(x_{Q_{M}}+x_{Q_{M-1}}\right)}=1
$$

Thus the wave function at $\Phi=2 \pi$ does not change under the set of transformations $p_{M}^{(f)} \rightarrow p_{M}^{(f)}+\pi$ and $p_{M-1}^{(f)} \rightarrow p_{M-1}^{(f)}+\pi$. Therefore the initial state and the final state are equivalent through the above set of the transformations. 


\section{APPENDIX C}

In this appendix, we show that the function (4.11) is odd function of $\Phi$. We start from the twisted boundary condition

$$
N p_{j}(\Phi)+\sum_{k=1(\neq j)}^{M} \theta\left(p_{j}(\Phi), p_{k}(\Phi)\right)=2 \pi I_{j}+\Phi
$$

where $N=2 M, I_{j}=\frac{2 j-M-1}{2}(j=1,2, \cdots, M)$ and

$$
\theta\left(p_{j}, p_{k}\right)=2 \arctan \left[\frac{\Delta \sin \left[\left(p_{j}-p_{k}\right) / 2\right]}{\cos \left[\left(p_{j}+p_{k}\right) / 2\right]-\Delta \cos \left[\left(p_{j}-p_{k}\right) / 2\right]}\right]
$$

By noting $\theta\left(p_{j}(\Phi), p_{k}(\Phi)\right)=-\theta\left(p_{k}(\Phi), p_{j}(\Phi)\right)$ and $I_{j}=-I_{M-j+1},(\mathrm{C} .1) \times(-1)$ is written as

$$
N\left(-p_{j}(\Phi)\right)+\sum_{j=1, k \neq j}^{M} \theta\left(-p_{j}(\Phi),-p_{k}(\Phi)\right)=2 \pi I_{M+1-j}-\Phi .
$$

Here we rewrite $n+1-j$ to $j$;

$$
N\left(-p_{M+1-j}(\Phi)\right)+\sum_{k=1(\neq M+1-j)}^{M} \theta\left(-p_{M+1-j}(\Phi),-p_{k}(\Phi)\right)=2 \pi I_{j}-\Phi
$$

Compare this and boundary condition at $(-\Phi)$

$$
N p_{j}(-\Phi)+\sum_{k=1(\neq j)}^{M} \theta\left(p_{j}(-\Phi), p_{k}(-\Phi)\right)=2 \pi I_{j}-\Phi
$$

we obtain

$$
p_{j}(-\Phi)=-p_{M+1-j}(\Phi) \text {. }
$$

Using this relation and $\epsilon\left(x_{b}-x_{a}\right)=-\epsilon\left(\left(-x_{a}\right)-\left(-x_{b}\right)\right)$ the wave function (1.3) 
becomes

$$
\begin{aligned}
& \chi\left(x_{1}, \cdots, x_{M} \mid \Phi\right) \\
= & \prod_{a<b} \epsilon\left(x_{b}-x_{a}\right) \sum_{Q}(-1)^{|Q|} \mathrm{e}^{i \sum x_{a} p_{Q_{a}}(\Phi)+\frac{i}{2} \sum_{a<b} \theta\left(p_{Q_{b}}(\Phi), p_{Q_{a}}(\Phi)\right) \epsilon\left(x_{b}-x_{a}\right)} \\
= & (-1)^{M(M-1) / 2} \prod_{a<b} \epsilon\left(\left(-x_{b}\right)-\left(-x_{a}\right)\right) \sum_{Q}(-1)^{|Q|} \mathrm{e}^{i \sum\left(-x_{a}\right) p_{M+1-Q_{a}}(-\Phi)} \\
& \times \exp \left[\frac{i}{2} \sum_{a<b} \theta\left(p_{M+1-Q_{b}}(-\Phi), p_{M+1-Q_{a}}(-\Phi)\right) \epsilon\left(\left(-x_{b}\right)-\left(-x_{a}\right)\right)\right] .
\end{aligned}
$$

Here we put $Q_{a}^{\prime} \equiv M+1-Q_{a}$, then this wave function is expressed as

$$
\begin{aligned}
& \chi\left(x_{1}, \cdots, x_{M} \mid \Phi\right) \\
= & \prod_{a<b} \epsilon\left(\left(-x_{b}\right)-\left(-x_{a}\right)\right) \sum_{Q^{\prime}}(-1)^{\left|Q^{\prime}\right|} \mathrm{e}^{i \sum\left(-x_{a}\right) p_{Q_{a}^{\prime}}(-\Phi)} \mathrm{e}^{\frac{i}{2} \sum_{a<b} \theta\left(p_{Q_{b}^{\prime}}(-\Phi), p_{Q_{a}^{\prime}}(-\Phi)\right) \epsilon\left(\left(-x_{b}\right)-\left(-x_{a}\right)\right)} \\
= & \chi\left(-x_{1}, \cdots,-x_{M} \mid-\Phi\right),
\end{aligned}
$$

where we used $(-1)^{|Q|}=(-1)^{\left|Q^{\prime}\right|} \times(-1)^{M(M-1) / 2}$. Therefore we get the relation

$$
\chi\left(x_{1}, \ldots, x_{M} \mid \Phi\right)=\chi\left(-x_{1}, \ldots,-x_{M} \mid-\Phi\right) .
$$

From this relation and $\left\{x_{j}\right\}=\left\{-x_{j}\right\}$ it is shown that the function (4.11) is odd function of $\Phi$;

$$
\begin{aligned}
& \sum_{\left\{x_{i}\right\}} \frac{\chi\left(x_{1}, \ldots, x_{M} \mid \Phi\right)^{*} \frac{\partial}{\partial \Phi} \chi\left(x_{1}, \ldots, x_{M} \mid \Phi\right)}{\left\|\chi\left(x_{1} \ldots, x_{M} \mid \Phi\right)\right\|^{2}} \\
= & \sum_{\left\{x_{i}\right\}} \frac{\chi\left(-x_{1}, \ldots,-x_{M} \mid-\Phi\right)^{*}\left(-\frac{\partial}{\partial(-\Phi)}\right) \chi\left(-x_{1}, \ldots,-x_{M} \mid-\Phi\right)}{\left\|\chi\left(-x_{1}, \ldots,-x_{M} \mid-\Phi\right)\right\|^{2}} \\
= & -\sum_{\left\{x_{i}\right\}} \frac{\chi\left(x_{1}, \ldots, x_{M} \mid-\Phi\right)^{*}\left(\frac{\partial}{\partial(-\Phi)}\right) \chi\left(x_{1}, \ldots, x_{M} \mid-\Phi\right)}{\left\|\chi\left(x_{1}, \ldots, x_{M} \mid-\Phi\right)\right\|^{2}} .
\end{aligned}
$$




\section{REFERENCES}

1. H. A. Bethe, Z. Physik71(1931) 205.

2. L. Hulthen, Ark. Mat. Astron. Fys. 26A11 (1938) 1.

3. C. N. Yang and C. P. Yang, Phys. Rev 147 (1966) 303; 150 (1966) 321, 327; 151 (1966) 258.

4. E. H. Lieb, Phys. Rev. 162 (1967) 162; Phys. Rev. Lett. 18 (1967) 1046; 19 (1967) 108.

5. M. Gaudin, Phys. Rev. Lett 26 (1971) 1301.

6. M. Takahashi, Prog. Theor. Phys. 50 (1973) 1519; 51 (1974) 1348.

7. V. E. Korepin, Commun. Math. Phys. 86(1982) 391.

8. F. Alcaraz, M. Barber and M. Batchelor, Phys. Rev. Lett. 58 (1987)771.

9. S.V. Pokorski and A.M. Tsvelick, Sov. Phys. JETP 66 (1987)1275.

10. V. Pasquier and H. Saleur, Nucl. Phys. B330 (1990) 523.

11. O. Foda and T. Miwa, Int. Journ. Mod. Phys.A7 1A(1992) 279.

12. B. Davies, O. Foda, M. Jimbo, T. Miwa and A. Nakayashiki, Commun. Math. Phys.151(1993) 89.

13. C. N. Yang, Phys. Rev. Lett. 19(1967)1312 ; Phys. Rev168(1968)1920.

14. R. J. Baxter, Ann. Phys. 58(1977)1395; Exactly Solved Models in Statistical Mechanics, Academic Press, New York (1982).

15. L.H. Takhtajan and L. D. Faddeev, Russ. Math. Survey 34(5) (1979) 11.

16. V. V. Bazhanov and N. Yu. Reshetikhin, Int. Journ. Mod. Phys. 4(1989) 115.

17. B. Sutherland and B. Shastry, Phys. Rev. Lett. 65 (1990) 1833.

18. V. E. Korepin and A. C. T. Wu, Int. J. Mod. Phys. B5 (1991) 497. 
19. N. Yu and M. Fowler, Phys. Rev. B46 (1992) 14583.

20. M. V. Berry, Proc. R. Soc. London A392 (1984) 45; see also, Geometric Phases in Physics, edited by A. Shapere and F. Wilczek (World Scientific, Singapore, 1989).

21. L.D. Faddeev and L.H. Takhtajan, Phys. Lett.85A (1981) 375.

22. F. C. Alcaraz, M. Barber and M. Batchelor, Ann. of Phys. 182(1988) 280. 